\title{
High-precision photometry with Ariel
}

\author{
Gyula M. Szabó ${ }^{1,2}$ (D) . Szilárd Kálmán ${ }^{1,2,3}$. Theodor Pribulla2,4 . \\ Antonio Claret ${ }^{5,6}$. Lorenzo V. Mugnai ${ }^{7}$. Enzo Pascale ${ }^{7}$. Dave Waltham ${ }^{8}$. \\ Luca Borsato $^{9} \cdot$ Zoltán Garai $^{2,4} \cdot$ Róbert Szabó $^{10,11,12}$
}

Received: 26 June 2020 / Accepted: 23 June 2021 / Published online: 5 August 2021

(C) The Author(s) 2021

\begin{abstract}
In this paper we describe the photometry instruments of Ariel, consisting of the VISPhot, FGS1 and FGS2 photometers in the visual and mid-IR wavelength. These photometers have their own cadence, which can be independent from each other and the cadence of the spectral instruments. Ariel will be capable to do high cadence and high precision photometry in independent bands. There is also a possibility for synthetic $J_{\text {synth }}, H_{\text {synth }}$, and wide-band thermal infrared photometry from spectroscopic data. Although the cadence of the synthetic bands will be identical to that of the spectrographs, the precision of synthetic photometry in the suggested synthetic bands will be at least as precise as the optical data. We present the accuracy of these instruments. We also review selected fields of new science which will be opened up by the possibility of high cadence multiband space photometry, including stellar rotation, spin-orbit misalignment, orbital precession, planetary rotation and oblateness, tidal distortions, rings, and moons.
\end{abstract}

Keywords Instrumentation - techniques: photometric

\section{Introduction}

High precision photometry of exoplanets opened a rich collection of tools for measuring the fundamental planet properties and to derive constraints for their internal composition and the atmosphere $[6,19,20,30,33,37,85]$. The transits of the largest planets could have been detected from the ground [8, 65], while space telescopes CoRoT [7], Kepler [13], TESS [67] and CHEOPS [12] allowed to overcome the atmosphere-limited photometry, leading to the discovery of a huge set of exoplanets.

Gyula M. Szabó

szgy@gothard.hu

Extended author information available on the last page of the article. 
The transiting planet moves along the transit chord, and selectively occults a part of the star along the path $[54,62,72,90]$. The precise shape of the resulting light curve depends on the optical interaction of the stellar surface and the planet's shape. The planet is usually characterised by a set of 5 parameters, such as the rotational period $(P)$, the transit time $\left(T_{0}\right)$, the planet-to-star radius ratio $\left(R_{p} / R_{*}\right)$, the relative semi-major axis $\left(a / R_{*}\right)$, and the impact parameter $(b)$ which is the distance of the center of the transit chord from the center of the star, in relative units. The star is described by the wavelength-dependent limb darkening coefficients [27-29]. In the case of rapidly rotating stars, the gravity darkening introduces a temperature gradient along the meridian, which has to be accounted for in such cases [2, 9, 26, 53, 79]. Most recently, stellar surface structures have been also involved to represent the presence of large stellar spots or faculae, and chromatic stellar activity features [68, $70]$.

Inferring planet parameters from a monochromatic photometry faces several important degeneracies in the parameter space. The most important of these are the multilateral degenerations of the limb darkening coefficients with $b$, and consequently, with $a / R_{*}$ and $R_{p} / R_{*}$ [36]. These degeneracies can be mostly resolved multicolour photometry and a direct fitting of the limb darkening coefficients from the multicolour observations [36].

The importance of precise exoplanet photometry from space is inevitably enormous. In the field, the precise photometry of Ariel (Atmospheric Remote sensing Infrared Exoplanet Large survey; [63, 64, 66, 84] will play a role as the first space observatory for exoplanets, which takes simultaneous multicolour photometry in three optical bands, together with the spectroscopic observations. The spectral range covers the visible from $0.5 \mu \mathrm{m}$ to the infrared to $7.8 \mu \mathrm{m}$ wavelengths $[38,63,84$, 91]. The multiband photometry opens new, mostly unexperienced opportunities in defining the science case of the Ariel mission. The role of the present paper is to 1) predict the precision in planet parameter determination for various planets around various stellar types; and 2) to explore new science that can be reached with help of the forthcoming multiband Ariel photometric data.

According to this dual aim, this paper consists of two major parts. It first gives a description of the photometric instruments of Ariel, from a viewpoint of the further data analysis and precision. After this review, a couple of new science cases are investigated for their feasibility with Ariel, and finally we conclude about the expected performance of these "exotic" observations.

\section{The photometric system}

The Ariel satellite mount two spectrometers and three photometers which will be the source of photometry data [59]. The Ariel Infrared Spectrometer (AIRS) is a two prism-dispersed channels that cover the band width (1.95-3.9) $\mu \mathrm{m}$ with $R>100$ and (3.9-7.8) $\mu \mathrm{m}$ with $R>30$ ). NIRSpec is slitless prism spectrometer with spectral resolving power $R>15$ in spectral range of (1.1-1.92) $\mu \mathrm{m}$. 
The Ariel photometric system contains three photometers, covering the $(0.5-$ 1.1) $\mu \mathrm{m}$ wavelength range in the visual and mid-IR. The photometers contain VISPhot $(0.5-0.6 \mu \mathrm{m})$ and two Ariel Fine Guidance Sensors (FGS1 at $(0.6-0.8) \mu \mathrm{m}$ and FGS2 at $(0.8-1.11) \mu \mathrm{m})$. These are wide band detectors, roughly corresponding to the Cousins $V$ (VISPhot), $R-I$ (FGS1), and SDSS $z$ (FGS2) passbands. The signal of these photometers can be read out independently from each other and the spectroscopic detectors. There is a possibility for a read-out cadence below 1 frame/second. It is also possible to read out one instrument at high cadence for rapid time resolution, and the other two instruments at a longer cadence, high $\mathrm{S} / \mathrm{N}$ mode. See the passbands in Table 1.

The NIRSpec low-dispersion spectrograph covers the (1.24-1.92) $\mu \mathrm{m}$ wavelength range. The NIRSpec spectra can also be used for photometry. The cadence of the NIR photometry will be identical to what is defined by the spectroscopic aims. The special advantage in the NIR range is, however, the possibility of defining custom digital passbands, either for dedicated spectro-photometric or wide-band photometry applications. Of these, a synthetic $J$ and $H$ band is of special importance, due to their widespread photometric applications. There will be a possibility to define further synthetic passbands in the thermal wavelengths from the AIRS instrument. This definition is flexible, as the response curve will be a set of weights that define a weighted mean of flux, centered at a weighted mean of wavelengths. Once the spectrum of a certain star and a planet is taken, the set of weights can be optimised to maximise the signal from the star, the signal from the planet, and to separate the two sources as much as possible. Now we just wish to qualitatively demonstrate the abilities of AIRS as a very wide-band photometer, and for this purpose we consider a Thermal wide band that sums up the entire signal detected by the AIRS..

Photometric and spectroscopic measurements will be taken simultaneously, with application of beam splitters. There will be a precise photometry for all spectroscopy targets. By optimising the observation strategy for transit time, duration and/or depth, we can measure transit time variations (TTVs), transit duration variations (TDVs) and transit depth variations very precisely.

Table 1 The visual photometric bands and synthetic NIR bands of Ariel

\begin{tabular}{llllll}
\hline Name & $\begin{array}{l}\text { Wavelength } \\
\mu \mathrm{m}\end{array}$ & $\begin{array}{l}\text { Bandwidth } \\
\mu \mathrm{m}\end{array}$ & $\begin{array}{l}\text { Left bin edge } \\
\mu \mathrm{m}\end{array}$ & $\begin{array}{l}\text { RightBinEdge } \\
\mu \mathrm{m}\end{array}$ & Instrument \\
\hline VISPhot & 0.55 & 0.1 & 0.5 & 0.6 & VISphot \\
FGS1 & 0.7 & 0.2 & 0.6 & 0.8 & FGS1 \\
FGS2 & 0.95 & 0.3 & 0.8 & 1.1 & FGS2 \\
$\mathrm{J}_{\text {synth }}$ & 1.26 & 0.16 & 1.18 & 1.34 & NIRSpec \\
$\mathrm{H}_{\text {synth }}$ & 1.65 & 0.28 & 1.51 & 1.79 & NIRSpec \\
\hline
\end{tabular}


Ariel will be the first dedicated exoplanet space telescope that is able to perform a multi-band photometry with ultra-short cadence (super-Hz sampling), paving a road to new science applications.

\subsection{Photometric performance}

To describe the performance of the Ariel photometers, we designed a set of 120 model systems, consisting of 3 representative kind of planets around 10 model stars, in all combinations at 4 different distances. The stars represented the A0, A5, F0, F5, G0, G2, G5, K0, K5, M0 spectral types, with solar metallicity and 65 million years of age. The stars were described by the $K$ absolute brightness, mass, radius (thus $\log g$ ), and temperature, which were taken from the Padova isochrones. The stars were placed at 10,30,100, and $300 \mathrm{pc}$ distances, assuming no interstellar absorption. Because of the spectroscopic observation requirements, only those configuration were taken into account where the host star had a $K$ magnitude in the range of (3.5-8.5) $\mu \mathrm{m}$.

Three model planets were assumed in these systems: a Hot Jupiter $\left(R_{p}=1.0 R_{J}\right)$, a hot Neptune $\left(R_{p}=3.9 R_{E}\right)$ and a hot super-Earth $\left(R_{p}=1.8 R_{E}\right)$. The model planets were placed on orbits that had a period of 5 days (hence, the semi-major axis depended on the stellar mass). The impact parameter was chosen to be $b=0$ in all cases.

$\mathrm{S} / \mathrm{N}$ ratios were calculated with ExoSim [70] for the three photometers and the individual dispersion elements in the spectrographs. Signal-to-noise ratios in the synthetic visual, near-infrared (NIR), $J, H$ and wide-band thermal bands were calculated by summing up the signal and the noise levels assuming independence of the noise in the different dispersion elements (white Gaussian noise). The summary of noise calculations are provided in an on-line data table.

The precise determination of the fundamental transit parameters such as transit time, transit duration and transit depth is the key to studies of planet structure, planet-planet perturbations, and the possible existence of a moon. To demonstrate the precision, we defined a set of template systems at various distances.

\subsection{Limb darkening coefficients}

For the light curve simulations, limb-darkening coefficients (LDC) were computed specifically for the Ariel photometric system and were performed by adopting the least-square method. [23] found that the law that best represents the profile of the specific intensities was the polynomial with 4-terms. Especially for transit photometry, the logarithmic, square-root, and non-linear laws are superior to the quadratic and linear laws [41]. Therefore we calculated four-parameter non-linear LDCs for a wide range of stellar parameters $\left(T_{\text {eff }}:(3500-40,000) \mathrm{K}, \log g:(0.0-+5.0), Z:(-5.0-\right.$ +1 ), and microturbulent velocities of $0,1,2,4$, and $8 \mathrm{~km} / \mathrm{s}$ ) according to the method described in details in [26] and [27]. The coefficients were calculated using ATLAS models.

The LDC law adopted in the simulations is written as:

$$
I(\mu) I(1)=1-c_{1}\left(1-\mu^{1 / 2}\right)-c_{2}(1-\mu)-c_{3}\left(1-\mu^{3 / 2}\right)-c_{4}\left(1-\mu^{2}\right) .
$$


Here, $I(1)$ is the specific intensity at the centre of the disk and $c_{n}$ coefficients are the corresponding LDCs. The $\mu$ is given by $\cos (\gamma)$, where $\gamma$ is the angle between the line of sight and the outward surface normal. Since the filter transmission curves for Ariel may change in the future, the model atmosphere intensities were convolved with a box-like transmission curve, covering the designed bands. This approximation is sufficient for feasibility analysis. When the filters will have been produced, the calculations will be updated accordingly, and extended to linear, square root, logarithmic and four-term models to support precise parameter determinations from Ariel photometric data.

These LDCs are not intended to serve as "official" Ariel LDCs, but support the repeatability and improvement of the results shown in this paper, and may be for calculating other scenarios. It is also known that a discrepancy between LD parametric models and reconstructions from transit photometry is usually experienced [46, 55, 58], and for quality data, the best approach is to let the LDCs be free in the fitting procedure[36, 40]. The present LDCs are however the best descriptions of the expected stellar profiles, and they can be widely used for various simulated observations for feasibility studies and also, target selections and updates. The complete data tables can be downloaded as an on-line source belonging to this paper.

\subsection{Precision of planet parameters}

The simulations were calculated with the FITSH code of [62]. The model light curves had a transit time of $T=0$, an orbital period of 5 days, while the semi-major axis was calculated from Kepler's 3rd law. White noise taken from ExoSim [70] was added to the light curves. Finally, these simulations reflected the constant host stars with the effective temperatures and $K$ magnitudes belonging to the modelled central star type and its assumed distance. For each light curves, 100 different realisations of the white noise were considered. Then the noisified synthetic light curves, as simulated observations, were fed again into FITSH in the solver mode. Although FITSH is capable to fit any kind of systematic patterns in a parametric analytical form and/or fed into the algorithm as detrending covectors, we did not use these options in solving the synthetic observations because no red noise nor slow varying systematics were added to the noise model. (This can be done later when the red noise model for the instruments will be available.) The resolver optimised the model parameters along an MCMC grid, then the errors in the parameters were characterised by the statistical standard deviation of the best-fit parameters.

Such way, the synthetic light curves of the 120 sample systems were solved to derive the precision of the best-fit planetary parameters. In Fig. 1 we show the resulting precision. The left column of figures shows the expected error in transit depth (and also $r_{p}^{2}$ if the stellar radius is exactly known) in percent. The right column is the expected error in transit mid-time in seconds. The first row of figures shows the precision of the parameters in case of the model Jupiter, the second one is the model Neptune, and the third one is the model super-Earth.

The curves of different colours show model stars at different distances. There are two curves in all colour, the one with circle symbols is VISPhot, the curve with triangle plot symbols show FGS2. FGS1 was calculated but not plotted to avoid the visual 
Jupiters

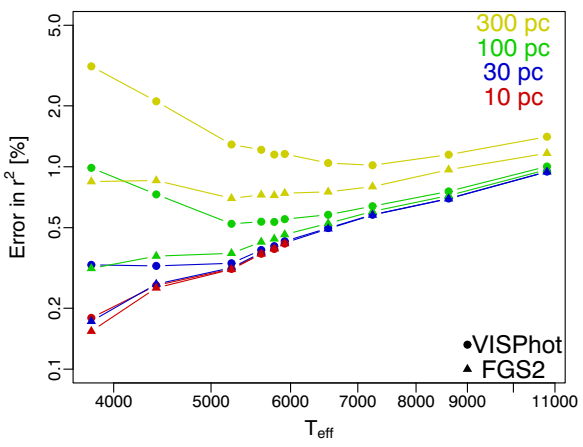

Neptunes

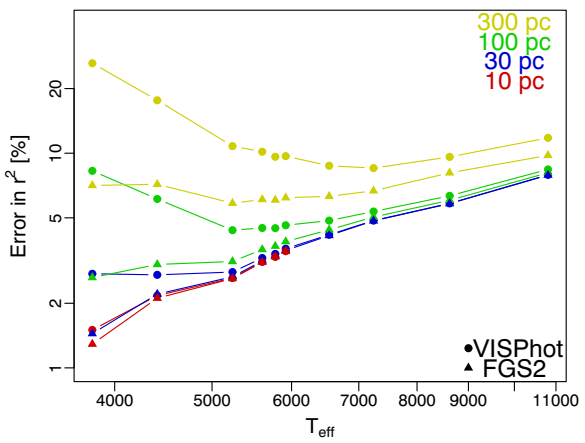

Super Earths

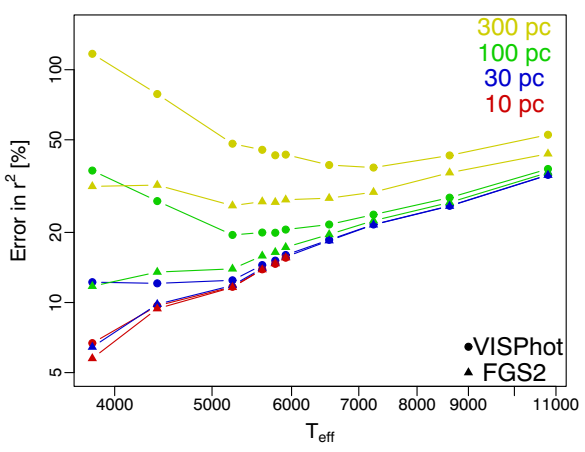

Jupiters

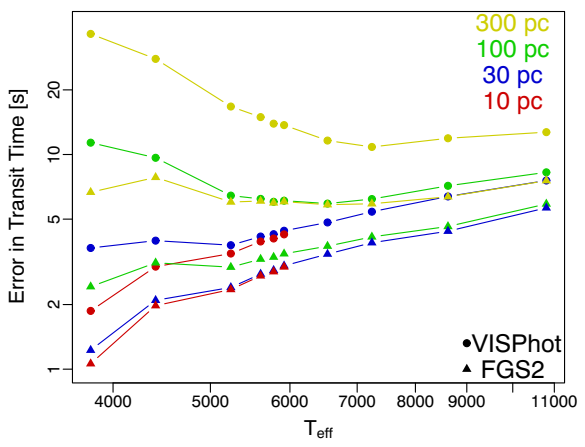

Neptunes

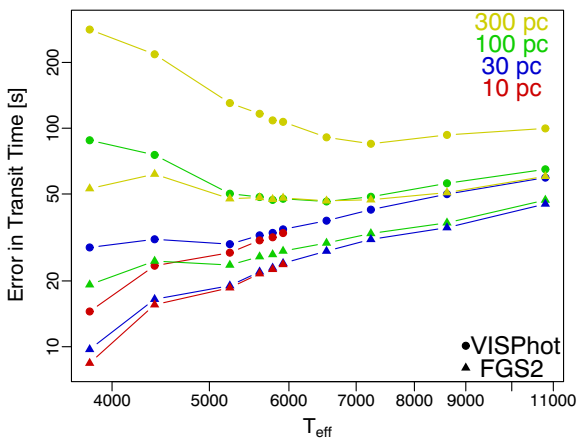

Super Earths

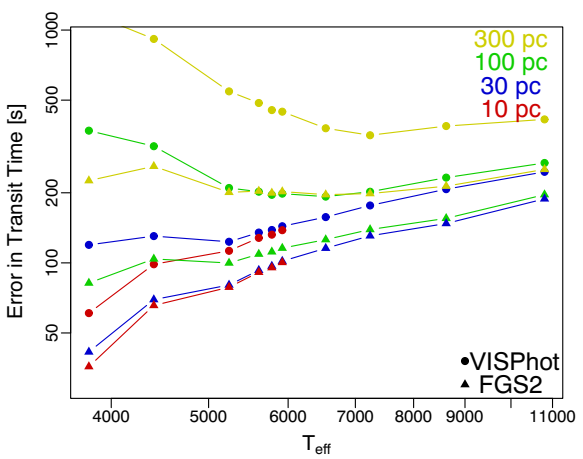

Fig. 1 The precision in transit depth and transit time around A0-M0 ZAMS stars, with various sized companions on 5 day orbit, as determined from VISPhot and FGS2 data

confusion. We checked that in all cases, the curve belonging to FGS1 is roughly halfway between VISPhot and FGS2.

Nearby early type (F0 and hotter stars at $10 \mathrm{pc}$ distance) are too bright, and no calculations were made - this explains the lack of red coloured points above 6000 
K effective temperature. Early type stars at the $30 \mathrm{pc}$ distance are still too bright in FGS2 band, and only VISPhot calculations were performed.

Nearby stars are all bright enough for a practically photon-noise free photometry, since the measurement will be limited by the $20 \mathrm{ppm} \sqrt{h r}$ instrumental noise floor, independently from the brightness of the star. In this case, the precision will be scaled by the depth and the duration of the transit signal. The smaller the star, the better precision in the planet parameters will be possible.

Towards the far end of the template star sample, late type stars are considerably fainter, and their measurement will be limited by the photon noise. Early type stars will be, however, still instrument-noise limited. There will be a local optimum for the precision in planet parameters, which is distance dependent. In summary, Ariel offers the most precise planet parameters in case of $\mathrm{K}-\mathrm{M}$ host stars nearby, $\mathrm{G}$ spectral type at $100 \mathrm{pc}$ distance, and $\mathrm{F}$ spectral type at $300 \mathrm{pc}$.

A general conclusion is that systems around the early type stars offer a very similar precision in the entire examined distance range, almost regardless to the distance. On the contrary, the precision of parameter determination in systems around late type stars cover a wide range: planets around nearby $M$ stars can be measured with the best precision in the sample, while M stars at $300 \mathrm{pc}$ distance give significantly less precision than all other model systems.

\subsection{Synthetic photometry from spectroscopy}

It is possible to define a synthetic $J_{\text {synth }}$ and $H_{\text {synth }}$ band from Ariel NIR spectrometer. These contain the sum of fluxes from dispersion elements centred between (1.181.30) $\mu \mathrm{m}$ ( $\left.J_{\text {synth }}\right)$ and (1.51-1.75) $\mu m$ ( $\left.H_{\text {synth }}\right)$. The advantage of these bands is that $H_{\text {synth }}$ has a better $\mathrm{S} / \mathrm{N}$ in all wavelengths as the visual band, while $J_{\text {synth }}$ has also a higher $\mathrm{S} / \mathrm{N}$ in most cases than the visual photometers, with the exception of $\mathrm{K}$ $\mathrm{M}$ stars. We compare the performance of these bands in Fig. 2 in the example of a Jupiter and a Neptune around A5, G0, K5 stars at 100 pc distance (all stellar and orbital parameters are identical to the calculations shown in Fig. 1.)

It is also possible to combine the signal from the three visual photometers, either by combining them into one band, or by solving the three-colour light curves jointly. This eventually leads to a better precision than the individual visual bands offer, the joint precision being compatible or better than $H_{\text {synth }}$. Also, the entire signal from the NIR spectrometer can be summed up or solved separately, dispersion-elementby-dispersion-element. This leads to the most precise parameter estimates, roughly 1.5 times more precise for transit depth and 1.8 times more precise for transit time (both factors slightly depending on the actual stellar parameters), with the longer cadence that will be determined by the observation requirements for the spectroscopic measurements. The thermal infrared synthetic band contains the entire signal from AIRS. This band is less precise than the combined NIR band, and the cadence will be also longer, so it has no added value to the planet parameter estimation. The thermal emission of the hottest planets will be visible in this band. Combining thermal and optical observations promises a possible measurement of the absorptivity on the 
A5 star with a Neptune at 100 pc

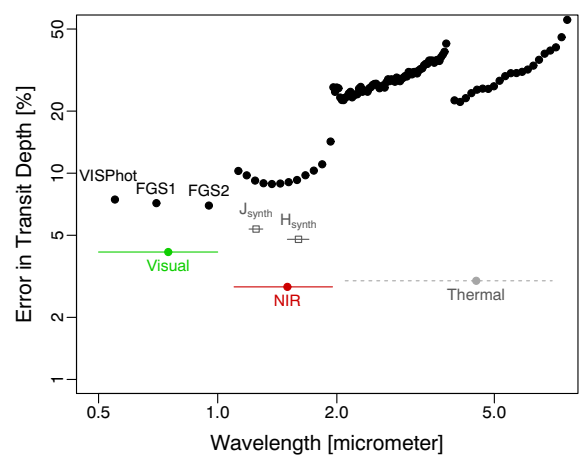

G0 star with a Neptune at 100 pc

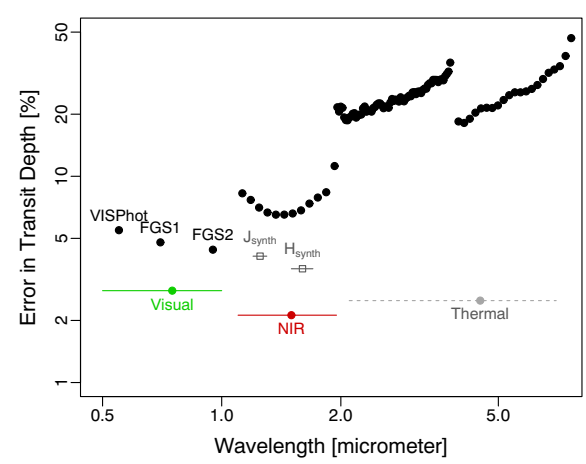

K5 star with a Neptune at 100 pc

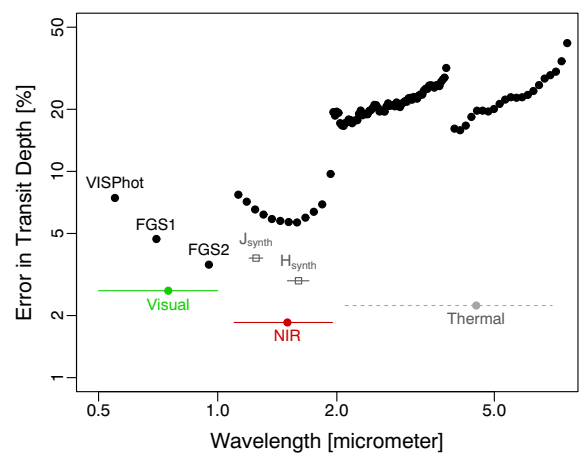

A5 star with a Neptune at 100 pc

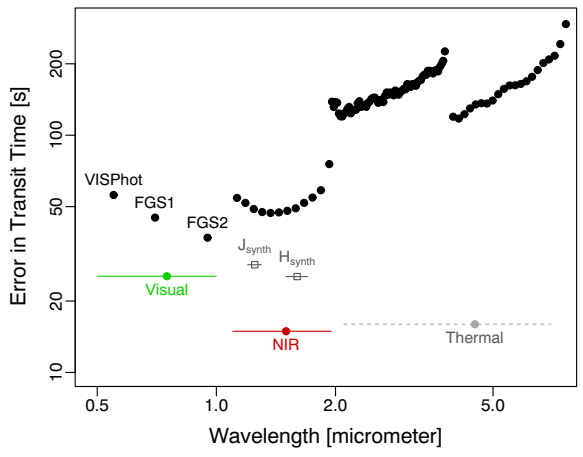

G0 star with a Neptune at 100 pc

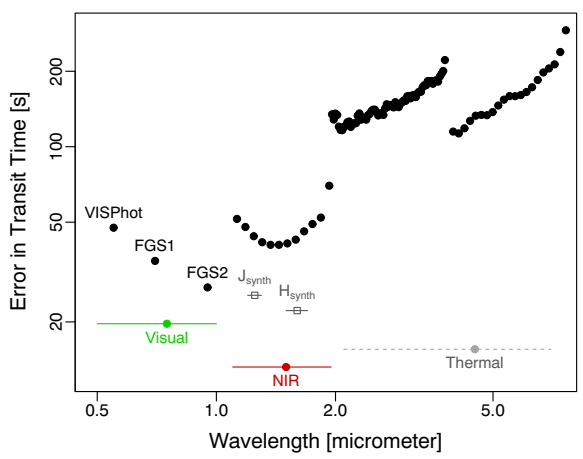

K5 star with a Neptune at 100 pc

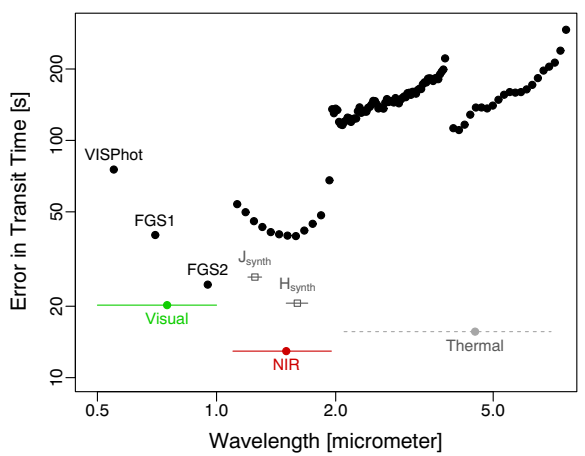

Fig. 2 The precision in transit depth (left column) and transit time (right column) around A0, G0 and K5 ZAMS stars, with a Neptune-sized companions on 5 day orbit. VISPhot, FGS1, FGS2 and unnotated black dots show the precision in a single dispersion element, for the full transit. $\mathrm{J}_{\text {synth }}$ and $\mathrm{H}_{\text {synth }}$ are synthetic $J$ and $H$ bands from NIR spectra. The precision in the combined Visual, NIR and Thermal infrared wavelengths are also indicated

surface of the planet ("albedo") in the wavelengths of the incident starlight and possibly, a large-scale mapping of the temperature distribution at the top of the atmosphere [53]. 


\section{Some aspects of new science with the Ariel photometric system}

In this Section, we discuss the feasibility of observing "exotic" light curve features beyond the classical 5-parametric reconstruction of transit data. The synthesis of the light curve based on 2D representation of the stellar disk, and distorted planets were sent into transit before this disk. The simulations were calculated in a $4000 \times 4000$ image matrix and with a stepsize of 15 seconds by default. The multiband signals then were compared to their 5-parametric reconstruction, with the following free parameters: transit time, orbital period, planet-to-star radius, orbital semi-major axis in units of stellar radius, impact parameter [61]. Since we included only single transits to the analysis, the orbital period was kept fixed at its nominal value (the input parameter of the simulations) and we fitted the remaining four parameters. We concluded about the observability of the tested case if the deviations exceeded the expected level of photon noise.

\subsection{Transit duration and transit depth variations - signs of orbit evolution}

As known from the theory of satellite motions [48], higher order moments of the gravitational potential of a host body yield periodic and secular perturbations in the orbits of nearby companions. The external gravitational potential of an extended body can be expressed as

$$
V(r, \theta)=-\frac{G M}{R_{\star}}\left[1-\sum_{n=2}^{\infty} J_{n}\left(\frac{R_{\star}}{r}\right)^{n} \mathcal{P}_{n}(\cos \theta)\right],
$$

where $M$ is the total mass, $R_{\star}$ is the equatorial radius, $J_{n}$ are constants and $\mathcal{P}_{n}$ are the Legendre polynomials. The most prominent perturbation is caused by $J_{2}$, due to the oblateness of the host body. MacCullagh's Theorem allows us compute $J_{2}$ using

$$
J_{2}=\frac{1}{M R_{\star}^{2}}\left(\Theta_{z z}-\frac{\Theta_{x x}+\Theta_{y y}}{2}\right)=\frac{\Theta_{z z}-\Theta_{x x}}{M R_{\star}^{2}},
$$

where $\Theta_{x x}=\Theta_{y y} \leq \Theta_{z z}$ are the principal moments of inertia. It is known that a non-zero $J_{2}$ results in secular perturbations in the angular orbital elements. Namely, the secular term in $\Omega$ (argument of ascending node) is computed $[48,80]$ as

$$
\frac{\mathrm{d} \Omega}{\mathrm{d} t}=-\frac{3}{2} J_{2} n\left(\frac{a}{R_{\star}}\right)^{-2} \frac{\cos \varphi}{\left(1-e^{2}\right)^{2}} .
$$

Here $n$ denotes the orbital mean motion, $a$ is the semi-major axis, $e$ is the orbital eccentricity, $i$ is the inclination of the orbit in reference to the sky plane, and $\varphi$ is the mutual inclination. 
Assuming a circular orbit for the transiting companion and by taking $\omega_{0}=d \Omega / d t$ as the precession rate inducted by $J_{2}$, we finally obtain

$$
\begin{aligned}
\frac{\mathrm{d} \cos i}{\mathrm{~d} t}= & -\frac{3}{2} J_{2} n\left(\frac{a}{R_{\star}}\right)^{-2} \times \\
& \times\left(\cos i \cos i_{\mathrm{p}}+\sin i \sin i_{\mathrm{p}} \cos \Delta \Omega\right) \times \\
& \times \sin i \sin i_{\mathrm{p}} \sin \Delta \Omega,
\end{aligned}
$$

where $i_{\mathrm{p}}$ is the orbital inclination to the stellar equator. Since transits are observed, we can say here that $\cos i \ll \sin i \approx 1$. In addition, $b=\left(a / R_{\star}\right) \cos i$, thus the above equation can be rearranged to give $\dot{b}$ as

$$
\dot{b}=-\frac{3}{2} J_{2} n\left(\frac{a}{R_{\star}}\right)^{-1} \sin ^{2} i_{\mathrm{p}} \sin \lambda \cos \lambda,
$$

where $\lambda$ is the orbital misalignment in celestial projection. This way, the observed change in transit duration can be directly related to the drift in the $b$ impact parameter, which is the relative distance between the transit chord and the center of the stellar disk, so the central transit has an impact parameter of $b=0$, and the grazing transit can be described by $b=1$. If $b$ varies due to orbital precession, the transit duration will be scaled as $\sqrt{1-b^{2}}$. Also, the transit duration variations can be related to a drift in inclination, $i$, reflecting the mutual precession of the spin axis of the star, and the orbital plane of the planet, around the axis of total angular momentum, and uncovers the important stellar and planetary dynamical parameters.

The general problem of a precessing stellar spin and planet orbital plane is rather complex. In Fig. 3 we show four stages of such a scenario. Since the total angular momentum depends of the tensor of inertia of the host star and the planet's orbital parameters, the mutual angles between the axes of precession of the star and the orbital plane depend on all system parameters. Also, the transit light curve will be modulated by the pattern of gravity darkening, which also has an untrivial behaviour just as illustrated in Fig. 3. Since the stellar rotation leads to an oblateness of the star, the transit duration and possibly, its rate will also deviate from the canonical value in a special manner. The problem is complex, and a general solution is required to solve the light curves with planetary precession. By the launch of Ariel, the necessary formalism will be developed, also taking into account the multiband Ariel photometry and how it resolves the expectable degenerations in the light curve.

Kepler-13 [75, 79, 80], MASCARA 4b [3], and Kelt-9 [2] an outstanding examples for an orbital precession leading to TDVs in normal planets. In Fig. 4 upper panel, we plot the increasing transit duration of Kepler-13Ab. Kepler and TESS data reveal significant transit duration variations. We see no TTV in the lower panel, proving that the reason of the transit duration variation is the stellar rotation and the orbital precession, and not and outer perturber.

By the time of Ariel observations, due to the observation window of roughly two decades, we expected to see a changing velocity, too. The current best realistic fits just goes through the TESS data point, and suggest a $\mathrm{d} b / \mathrm{d} t$ value of 0.13 , predicting a roughly central transit by the time of Ariel observations. Later on, 80 years from 
Fig. 3 Rapid stellar rotation leads to the precession of the stellar spin axis and the orbital plane. This leads to transit depth variation and possibly complex light curve distortions due to the changing pattern of gravity darkening along the transit chord
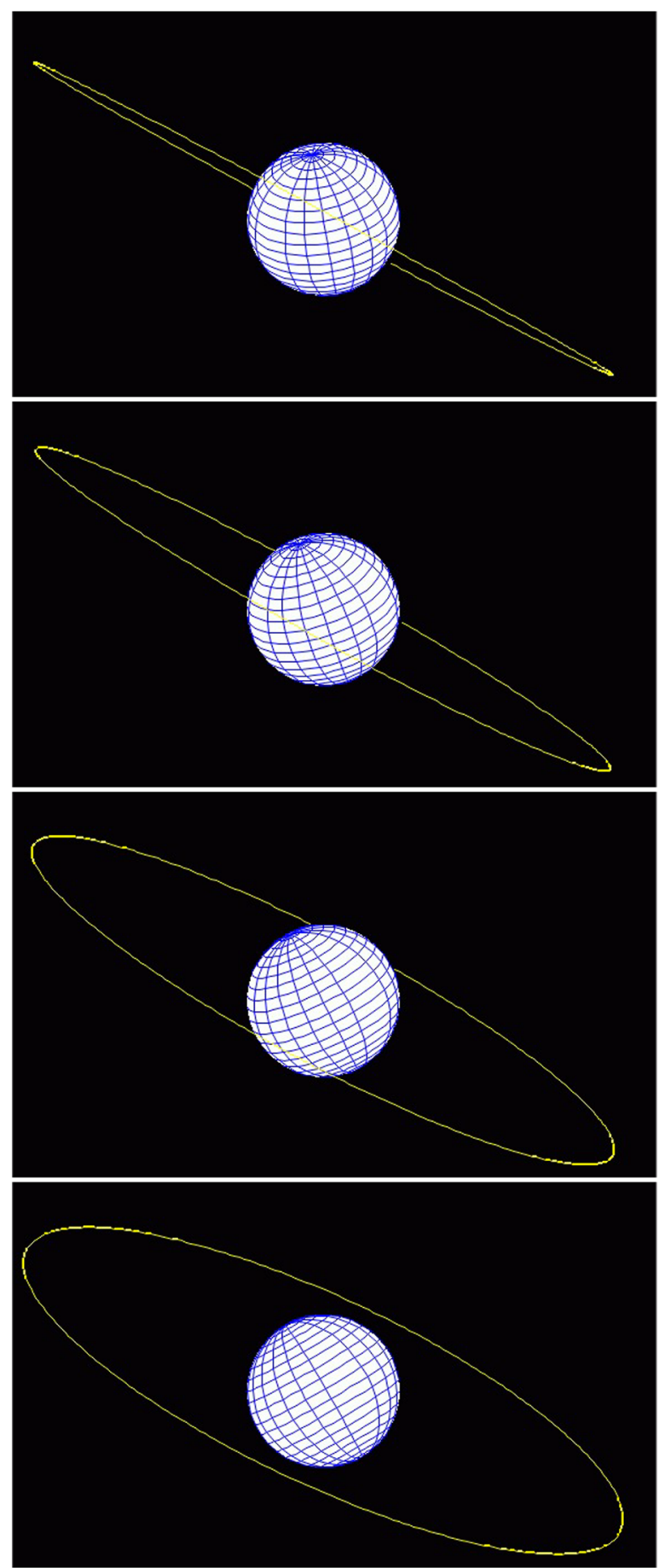

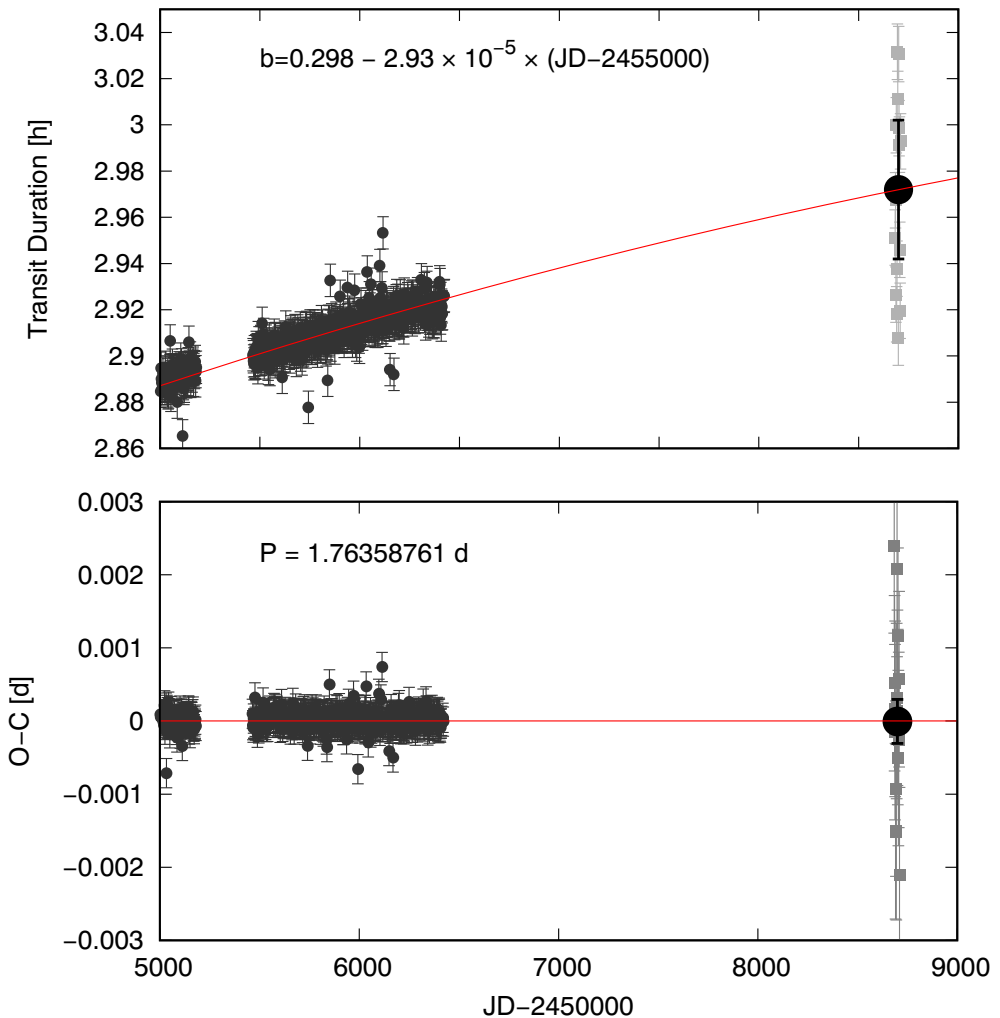

Fig. 4 Transit duration of Kepler-13.01. Duration data from Kepler photometry are plotted individually for all transits in Q2-Q17, TESS data were fitted in the folded transit light curve (Szabó et al. 2020)

now, Kepler-13Ab evolves to a non-transiting planet, similar to the scenario shown in Fig. 3.

\subsection{Rapidly rotating stars as planet hosts}

Rapid rotation is typical for single main-sequence stars earlier than about F5 (socalled Kraft break, [52]) due to the lack of the convective envelopes. The rotation rate is a function of mass and evolutionary state so it is routinely used in gyrochronology (see e.g., [87]).

The rapid rotation of early-type stars coupled with the lack of strong spectral lines other than Hydrogen Balmer lines limits radial-velocity measurement precision [43]. Thus for many exoplanets detected by the transit method, only the upper mass limit from spectroscopy is available (e.g. the case of XO-06b, [35]).

Another consequence of the rapid rotation is the gravitational darkening first described for radiative envelopes by [88]. The gravitational darkening is the result of the different energy transfer rate across a rotating star due to the local gravity differences. Because the local gravity is largest at the poles, they are the hottest places 
on rapid rotators. Recently, an analytical method producing very accurate results especially for fast rotators was published by [39]. The conditions of the von Zeipel theorem also fail for cooler stars [22, 24, 25]. The valid method has the following properties: a) to be applied to convective and/or radiative envelopes; b) one can investigate the influence of the optical depth in the GDE by changing the fitting point to impose the boundary conditions, without loss of generality; c) The GDE can be computed as a function of initial mass, chemical composition, evolutionary stage and other ingredients of input physics. More realistic atmosphere models can be easily incorporated as external boundary condition, as done in [25].

The rotation also modifies the shape of a star making it oblate. When the rotation rate reaches so called break-up velocity (the equator gravity is zero), the ratio of the equatorial to polar radius is 3:2. This is valid in so-called Roche approximation of the surface, when the star is assumed to be a mass point [69].

Alhough the fast rotation complicates the transit light-curve analysis [9], it enables to constrain the orientation of the rotational axis with respect to the orbital plane if a high-precision space photometry is available [11, 81]. The fast rotation also enables to directly detect the planet feature in mean line profiles (see e.g. the case of WASP$33 \mathrm{~b},[31])$ and more recently to detect the nodal precession of the exoplanet orbit spectroscopically [89].

Unlike in slowly rotating late-type stars, where the transit depth primarily depends on the ratio of the planet and stellar radii, for rapid rotators the impact factor and stellar spin-orbital plane misalignment significantly affect the transit depth. Spin-orbit misalignment in rapid rotators causes asymmetric transit light curves. Multi-colour light curves are critical to disentangle the effects of the limb and gravity darkening. With high-precision multi-band light curves and spectroscopically determined projected misalignment angle $\lambda$, it is in principle possible to better constrain the orbit of the planet, to determine the obliquity of the stellar rotational axis, and to lift the parameter degeneracies.

The synthetic light curves in VISPhot, FGS1 and FGS2 were computed (for details see [81]) for an extreme case of Kelt-21b [47], which has projected rotational velocity of $v \sin i=146 \mathrm{~km} / \mathrm{s}$. Although the projected spin-axis orbital plane misalignment is only $\lambda=-5.6$ degrees, the rapid rotation strongly deforms the transit light curve. Moreover, the large surface temperature gradients across the surface of the parent star result in significant differences between the photometric bands (see Fig. 5).

Even if the spin axis of the star is perfectly aligned, the effects of gravitational darkening due to the rapid rotation are significant. The brightness drop due to a transiting planet strongly depends on the transit impact factor. The synthetic light curves for the case of Kelt-21b with $\lambda=0$ degrees are shown in the bottom panel of Fig. 5. The principal difference is the transit amplitude depending strongly on the wavelength of observation.

\subsection{Oblateness of fast rotating planets}

Most exoplanets are known to be in a bound spin-orbit state due to their vicinity to the central stars and the tidal evolution. However, farther gas giants in the Solar 

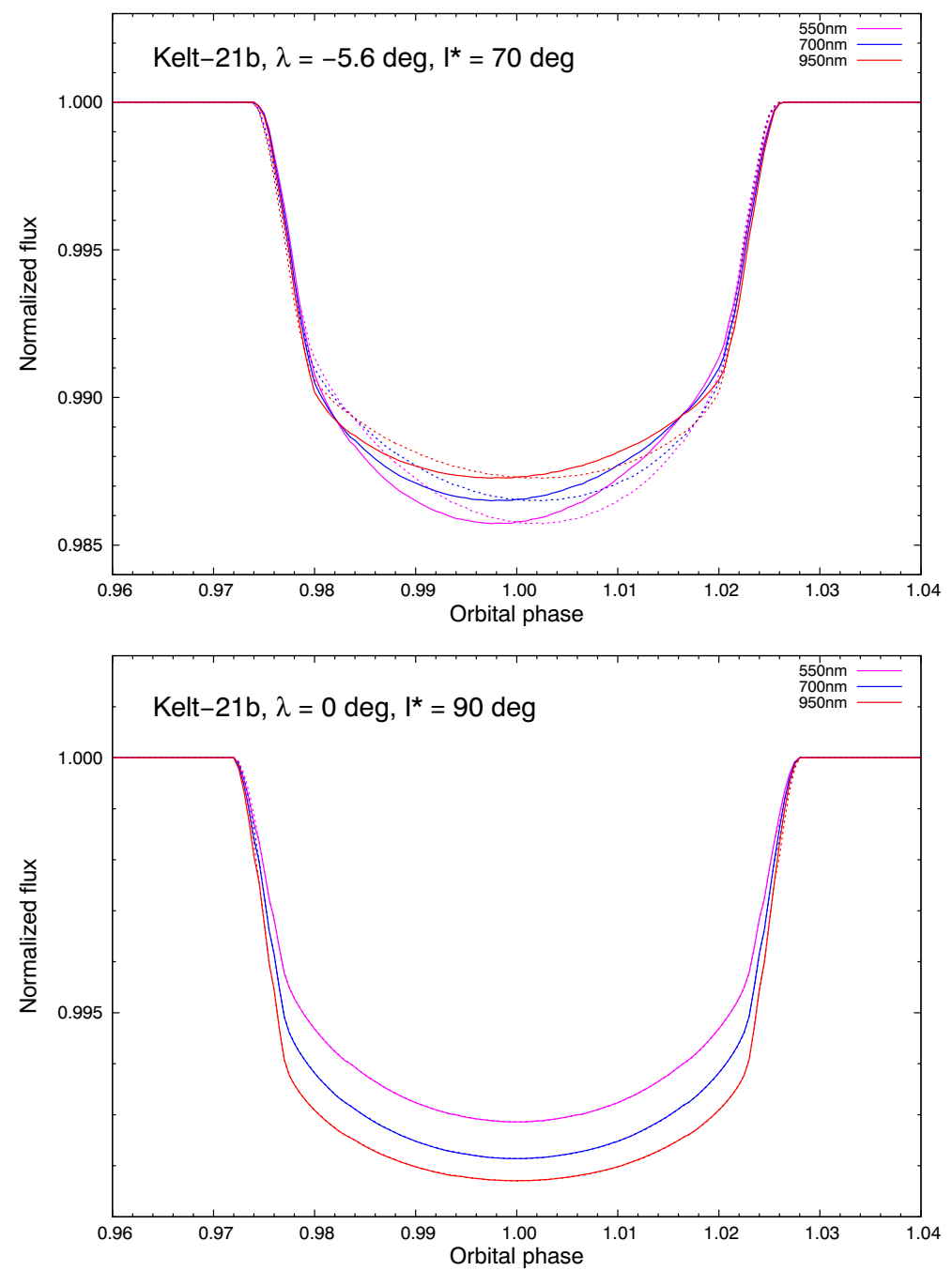

Fig. 5 Synthetic light curves of Kelt-21b for three photometric bands centered at $550 \mathrm{~nm}, 700 \mathrm{~nm}, 950$ $\mathrm{nm}$. The projected rotational velocity of the parent star is $146 \mathrm{~km} / \mathrm{s}$. Top: The stellar spin axis obliquity is assumed to be $70^{\circ}$, the projected spin axis-orbital plane misalignment is $\lambda=-5.6^{\circ}$. The dashed curves are phase-mirrored original curves to highlight the light-curve asymmetry. Bottom: The stellar spin axis obliquity is assumed to be $90^{\circ}$, the projected spin axis-orbital plane misalignment is $\lambda=0^{\circ}$

System are known to have an oblate planetary body, Saturn being the most oblate example. Ariel will be able to detect the rotation of the fast rotating exoplanets, via their oblateness induced by the rotation.

The detection of planet rotation can be identified as certain light curve anomalies which are most prominent at the ingress and egress phases [10,73]. The detection must be a difficult task due to the very low $\mathrm{S} / \mathrm{N}$ ratio. If there is also planetary precession, the depth variations caused by a precessing oblate planet can be better identified 
[17]. Here we focus only on the detectability from one transit alone, since planet precession is a long-term effect and its modelling requires many model parameters. We show that Ariel may be able to detect the planetary rotation from single epoch measurements, making use of the multiband photometry.

Rotational oblateness can be quantified by the oblateness parameter $f$. This is related to the rotational rate of the planet and its mass. With simple dynamical reasoning we can write as:

$$
f=\frac{R_{E}-R_{P}}{R_{P}}=\frac{\Omega^{2} R_{E}^{3}}{G M},
$$

where $R_{E}$ and $R_{P}$ are the equatorial and polar radii, respectively, and $\Omega$ and $M$ are the angular velocity and mass of the planet (Murray \& Dermott 2000). This will result in slight differences in the observed light curve compared to that of a perfectly spherical object. Here follows a demonstration of what the multi-band photometry done with Ariel will be able to disclose.

We assumed a Sun-like central star, orbited by a giant planet that had some rotational oblateness (representing Saturn, and also a planet close to the rotational break-up velocity). We modelled 3 scenarios (Fig. 6, upper left panel):

- A: $f \sim 0.09, b=0$, and 0 inclination of the planetary axis;

- B: $f \sim 0.38, b=0.25$, and $30^{\circ}$ planetary inclination;

- $\quad$ C: $f \sim 0.09$ (similar to Saturn), $b=0.25$, and $30^{\circ}$ planetary inclination.

The results of these simulations are plotted (with dots) in the top right and two bottom panels of Fig. 6.

The simulated planetary transits were fitted using FITSH/lfit [61], as described in Section 3. The best-fit curves are overplotted in the top parts of the top right and bottom two panels of Fig. 6. Subtracting these from the original light curves yields the residuals shown in the bottom parts of said panels in Fig. 6, which show the light curve components of planetary oblateness in the photometry.

The spin-orbit misalignment of the B and $\mathrm{C}$ scenarios results in asymmetric light curves, and this asymmetry is clearly visible in the two bottom panels of Fig 6. Eventually, the light curves are symmetric for a symmetric geometrical configuration, and are very asymmetric if slight asymmetries occur in the geometry.

The amplitudes of the residuals near ingress and egress are $\sim 50 \mathrm{ppm}, \sim 70$ $\mathrm{ppm}$ and $\sim 300 \mathrm{ppm}$ for $\mathrm{A}, \mathrm{B}$ and $\mathrm{C}$ cases, respectively, meaning that with a 20 ppm precision, planetary oblateness can be recognised. This means that planets of the brightest Ariel target stars can be explored with expectations of a conclusive result. In the case of these stars, shot noise exceeds the photon noise, and the noise of data points will be the noise floor (expected to be around $20 \mathrm{ppm}$ ) regardless of the exposure time. Fig 6 shows that the time scale of the variation in the anomalies is in the order of 0.01 days. This means that those stars are promising for this measurement where an integration of 5-10 minutes gives a photon noise well below the $20 \mathrm{ppm}$ level and the observation will be shot-noise limited. This is fulfilled for stars brighter than $K \approx 8$ magnitudes, depending on the effective temperature, too. 

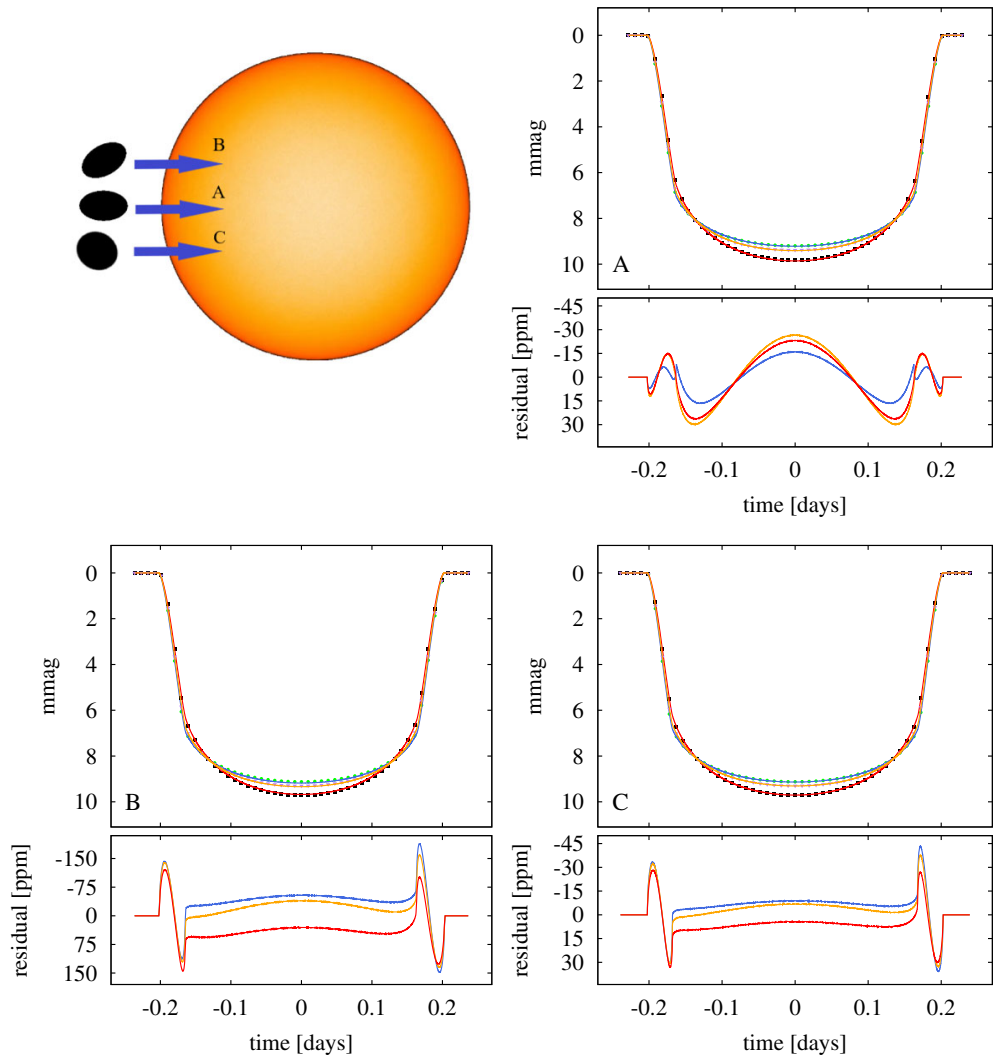

Fig. 6 VISPhot, FGS1 and FGS2 light curve models of planets with oblateness due to rapid rotation. The geometrical configurations are shown with the labels. The light curve residuals are calculated to the best-fit [61] solution. Panels A-C show a planet close to rotational break-up at central transit, the same planet on the $b=0.25$ transit chord and $30^{\circ}$ inclination of the planet's rotational axis, and a planet with a Saturn-like oblateness at $b=0.25$. Note the colour-dependent shift in the residuals close to the central position of the planet that breaks up the possible degenerations of planetary oblateness and the ambiguity in limb darkening coefficients

Also, during the transit, the mean value of the residuals are also colour-dependent. This colour bias can be measured with the precision of Ariel, and can be an evidence for a non-spherical planet shape, and hence, rapid planetary rotation.

\subsection{Rings around planets}

Rings around planets can be primordial or outcome of collisions of large moons, and in both cases, they can be places where newborn exomoons can also form [34]. The possible detection of the rings is due to revealing light curve anomalies, similarly to the oblate planets. These deviations are tiny in both cases, which makes the detection of rings a challenging task [86]. Besides transit depth variations, [92] suggested the detectability of photo-ring effects, connected to the scattering properties of the small 
grains that build up the ring. A specific tool for simulating planet light curves with a ring is SOAP3.0 [4] which is a numerical tool to simulate ringed planet transits and measure ring detectability based on amplitudes of the residuals between the ringed planet signal and best fit ringless model (Fig. 7).

We adapted our transit photometry simulator to the simulation of rings, and simulation of planet oblateness and rings together, too. It has to be emphasized that the significant difference between light curve distortions caused by planet oblateness or rings is that the different optical properties: shape distortions of the planet only hide certain segments of the stellar surface along they path, but grains can scatter the light to forward directions, too. This scattering depends on the size and the material properties of the grains, and the wavelength of the observation. The spectrum of the forward scattered light due to a ring may be observable in spectra, too, which also helps this distinction.

Here we intend to demonstrate the possibility of these kind of measurements with Ariel in a simplified example. For this reason, we simulated monochromatic light curves as observed in VISPhot, and neglected the forward scattering. (Examples of multiband transits with wavelength-dependent forward scattering is found in the paper by Garai et al. in this issue.)
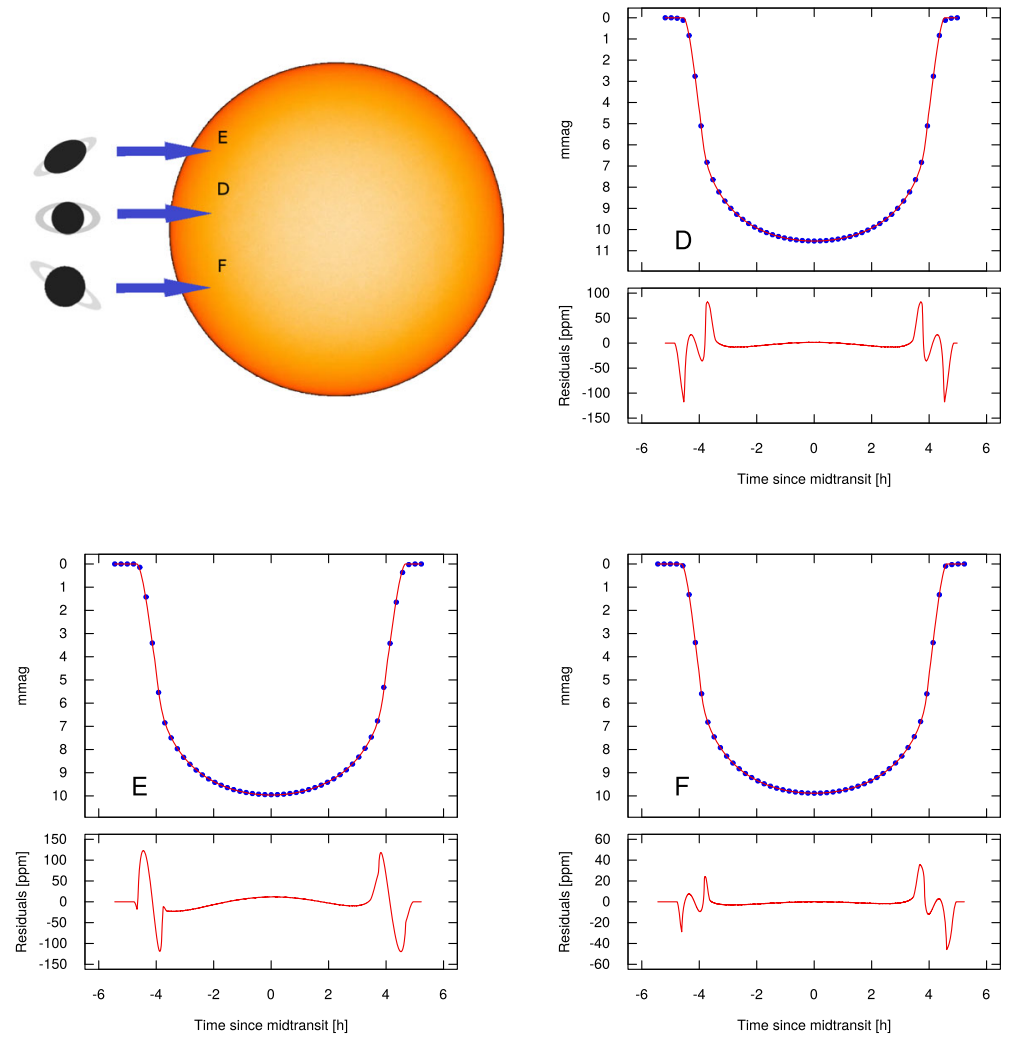

Fig. 7 The modelled scenarios, the resulting light curves and the residuals of the fit 
To describe a ringed exoplanet, we introduced the inner and outer radii of the ring (set to $1,5 R_{P}$ and $2 R_{P}$, respectively), the angle of its axis and the planetary orbit $(\varphi)$, and $\vartheta$, the projected tilt of the ring to the planet's orbital plane $\left(0^{\circ}\right.$ and $90^{\circ}$ for ring projection parallel and perpendicular to orbital plane respectively) The ring absorbed $10 \%$ of the incident light, and had no forward scattering. We modelled the following scenarios:

- $\quad \mathrm{D}: f=0, b=0, \vartheta=0^{\circ}, \varphi=60^{\circ}$;

- $\mathrm{E}: f \sim 0,38, b=0,25, \vartheta=30^{\circ}, \varphi=60^{\circ}$;

- $\mathrm{F}: f=0, b=0,25, \vartheta=30^{\circ}, \varphi=60^{\circ}$.

The resulting light curves are in good agreement with those described by Barnes and Fortney (2004) and Heising et al (2015). The similarities with the oblate cases are remarkable, especially for scenario E. This would mean that while we should be able to determine that an exoplanet has rings, based on the analysis shown above it would be difficult to determine whether a given light curve is a result of rings or oblateness alone, or the combination of these two effects.

\subsection{Phase curves and tidally deformed of planet}

In close-in systems, both planets and the stars are deformed due to the mutual gravitational tidal distortions. During the transit, the elongated shape of the planet can also be observed, as deviations from the circular planet silhouette. However, the shape of the actual deviations are different $[5,32]$.

Both the magnitude of this deviation and the chromatic effects are similar to the case of oblate planets, and the observability is similar to the case of planet rotation or planet rings. An important difference is that tidal deformations are the best observable in the case of the most close-in planets, while fast planet rotation is expected only far from the star. Since tidal deformation affects both the planet and the star, tidal effects in the transit light curve go hand in hand with phase curve variations ([42, 74]). The complete observation will be more complex and must be studied with complex tools that are to be developed in a future work.

\subsection{The quest for moons}

Regular moons are predicted to form generally in planetary systems, as a direct outcome of planet formation. During the core-accretion, gravitational perturbations between planet embryos imply a series of constructive impacts up to the formation of a fully grown planet [60]. This phase may happen between a few Myrs to a few 100 Myrs after the star formation (see e.g. [18]), and in these processes, satellites may form around the growing planets. Such giant impacts are advocated for the formation of the Earth's Moon [14] and for the formation of Uranus and Neptune's satellites [57]. Satellites may also be formed in rings, which could be natural outcomes of giant impacts or tidal disruptions, either in the planet formation phase or later in a relaxed planetary system (see e.g. [15, 21, 34]).

An alternative scenario invokes the accretion of moons in the gaseous circumplanetary envelopes that surround the most massive giant planets during their growth in 
the gaseous protoplanetary disks (see e.g. $[16,71]$ ). Inside the circumplanetary disk, the solid material is replenished by the surrounding protoplanetary disk. This solid material is thought to coagulate in the form of large satellites in a way similar to planets. Then the young satellites migrate inward, and sometimes sink into the planet. When the circumplanetary disk disappears, the satellite system is stabilized on the short term.

After the planets have been formed, the orbits of moons evolve due to the planet's tides. Tidal forces lead to the dissipation of energy. On one hand, this usually results in the gradual expansion of the moon's orbits, if the evolution starts outside the planet's synchronous radius. (A synchronous orbit is where the moon's orbital period is equal to the planet's rotational period.) On the other hand, moons inside the synchronous orbit will migrate inwards and can reach the planet's Roche limit where the moons are disrupted by tidal forces.

Transiting exoplanets that possess moons have transit times that depart from perfect regularity. Hence, detection of TTVs (transit time variations) may allow the identification of potential exomoon candidates [49, 50, 76-78, 82, 83]. TTVs alone are not sufficient to uniquely identify the presence of an exomoon because of possible perturbations by other bodies. Nevertheless, detecting TTVs will identify candidates meriting further investigation with additional techniques. With methods estimating the full photometric effect of the moon are more sensitive to the size of the moon, rather than to its mass [78], and a combination of the dynamical and photometric signal, the photodynamical method can reveal the full information, including the moon's mass [51]. However, instrument systematics still can mimic a presence of a moon $[44,82]$.

The most secure detection of a moon is observing a consistent combination of different light curve characteristics, including TTVs, TDVs, and most preferentially, a light curve distortion that is characteristic to a moon (Fig. 8). Capabilities of Ariel are promising here, since the systematics of the different instruments will be mostly uncorrelated, and biases due to long-lasting systematics can be filtered out. Also, the multiband photometry helps certifying that the biases in the three photometers and the infrared are really identical to a companion, possibly leading to a multispectral confirmation of the presence of the largest exomoons.

\subsection{Transit timing variations - mass and orbital parameters improvements}

The Transit Time Variation (TTV) technique is a powerful tool to discover and characterise exoplanetary systems by measuring changes in the orbital period due to gravitational interaction $[1,45,56]$. The fast-cadence photometric data provided by the Ariel Fine Guidance Sensors (FGS1 and FGS2), NIRSpec, and AIRS will allow us to measure the planetary transit time at precision and accuracy level of few seconds. This will also break the degeneracy on doubtful dynamical solutions by extending the temporal baseline of known planets in multiple-planet systems showing TTV signals. We present the Ariel timing performances based on simulations of real targets and some possible science cases in a separate paper (Borsato et al., 2020) in the present journal issue. 


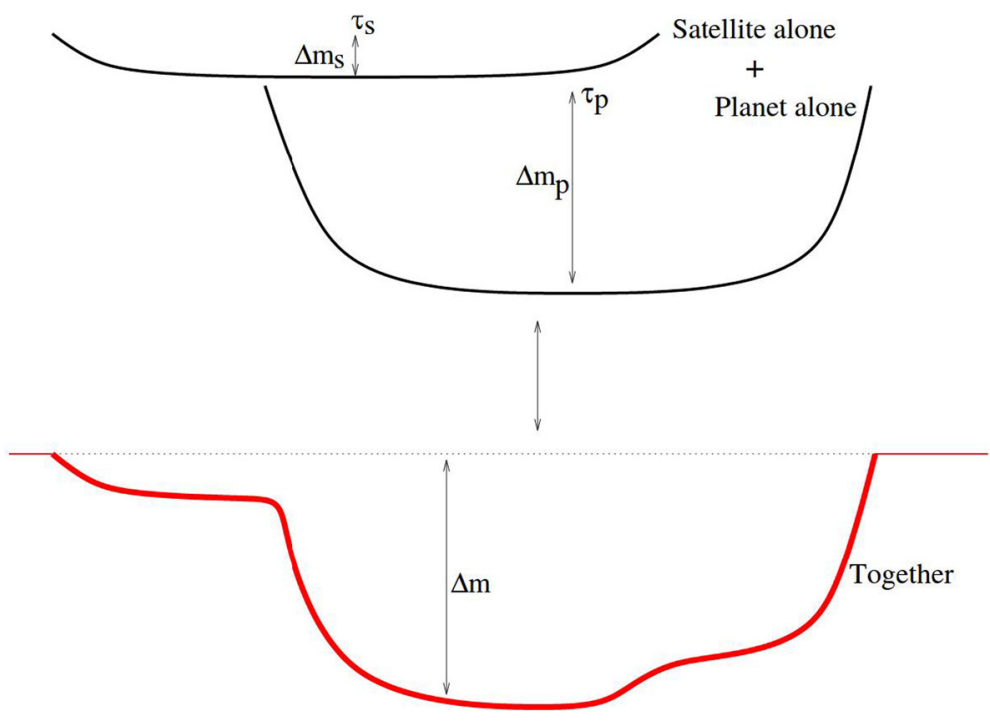

Fig. 8 Light curve of a planet+moon scenario is combination of the planet alone and the moon alone light curves. The precision required to directly observe the distortion caused by the moon is in the order of an observation of a planet of the same size [76]

\section{Summary}

In this paper, we gave a description of the Ariel photometric system, consisting the measurements of VISPhot, FGS1, FGS2 photometers and also synthetic photometry in $J_{\text {synth }}, H_{\text {synth }}$ and wide-band Thermal bands, derived from the signal of NIRSpec and AIRS spectra, in term of performance in planet parameter determination in a wide palette of stellar and planet parameters. We examined the feasibility of new science cases "beyond the five planet parameters", making use of the high precision multiband data and non-standard ways of modeling signals of non-spherical planets, and accounting for effects of stellar rotation.

The main conclusions can be summarised as follows.

- Timing precisions of 10-40 s are possible for typical stars in the Target list. The most precise band is $H_{\text {synth }}$, the most precise measurement is the wide-band NIR photometry directly from NIRSpec. Independent measurements from the photometer signal and the wide-band Thermal IR is possible, with only a bit less precision than in $H_{\text {synth }}$.

- TDV measurements can be as precise as 2-3\% in the planet radius parameter.

- Both transit timing and transit depth is most precise for stellar types close to the Sun. For earlier type stars the stellar radius compresses the signal, in case of later type stars the activity and the faintness of the star increase the noise.

- A joint analysis of TTVs and TDVs can lead to three-dimensional solutions for the planets' orbits and the rotation properties of the star.

- We expect that planet oblateness due to rapid planet rotation, tidal distortions, and the observation of rings around planets can also be observable in the case 
of the conceivable best examples. The distinction between these three sources of light curve distortion is more difficult, and requires the joint analysis of the multiband photometry.

- The quest for exomoons is most promising with combining several possible exomoon effects, including TTVs, TDVs, and mostpreferentially, a light curve distortion that is characteristic to a moon.

- The independence of instrument systematics in the different bands and the possibility of a simultaneous multiband signal modeling offers a better reconstruction of the transit signals.

- The examined cases illustrate that the multiband capabilities of Ariel highly support the observation (or possible discovery) of exotic planet physics.

Acknowledgements This work has been supported by the Hungarian National Research, Development and Innovation Office (NKFI) grants K-119517, K-115709, and GINOP-2.3.2-15-2016-00003, the Lendület Program of the Hungarian Academy of Sciences, project No. LP2018-7/2020, the MTA-ELTE Lendület Milky Way Research Group, and the City of Szombathely under agreement No. S-11-1027. L.V.M. and E.P. was supported by the ASI grant n. 2018.22.HH.O. ZG and TP acknowledge support from the VEGA grant of the Slovak Academy of Sciences No. 2/0031/18 and by the grant of the Slovak Research and Development Agency number APVV-15-0458. LBo acknowledges the funding support from Italian Space Agency (ASI) regulated by "Accordo ASI-INAF n. 2013-016-R.0 del 9 luglio 2013 e integrazione del 9 luglio 2015 CHEOPS Fasi A/B/C”.

Funding Open access funding provided by Eötvös Loránd University.

Open Access This article is licensed under a Creative Commons Attribution 4.0 International License, which permits use, sharing, adaptation, distribution and reproduction in any medium or format, as long as you give appropriate credit to the original author(s) and the source, provide a link to the Creative Commons licence, and indicate if changes were made. The images or other third party material in this article are included in the article's Creative Commons licence, unless indicated otherwise in a credit line to the material. If material is not included in the article's Creative Commons licence and your intended use is not permitted by statutory regulation or exceeds the permitted use, you will need to obtain permission directly from the copyright holder. To view a copy of this licence, visit http://creativecommons.org/licenses/by/4. $0 /$.

\section{References}

1. Agol, E., Steffen, J., Sari, R., Clarkson, W.: On detecting terrestrial planets with timing of giant planet transits. MNRAS 359(2), 567-579 (2005). https://doi.org/10.1111/j.1365-2966.2005.08922.x, arXiv:astro-ph/0412032

2. Ahlers, J.P., Johnson, M.C., Stassun, K.G., Colón, K.D., Barnes, J.W., Stevens, D.J., Beatty, T., Gaudi, B.S., Collins, K.A., Rodriguez, J.E., Ricker, G., Vanderspek, R., Latham, D., Seager, S., Winn, J., Jenkins, J.M., Caldwell, D.A., Goeke, R.F., Osborn, H.P., Paegert, M., Rowden, P., Tenenbaum, P.: KELT-9 b's Asymmetric TESS Transit Caused by Rapid Stellar Rotation and Spin-Orbit Misalignment. AJ 160(1), 4 (2020). https://doi.org/10.3847/1538-3881/ab8fa3, arXiv:2004.14812

3. Ahlers, J.P., Kruse, E., Colón, K.D., Dorval, P., Talens, G.J., Snellen, I., Albrecht, S., Otten, G., Ricker, G., Vanderspek, R., Latham, D., Seager, S., Winn, J., Jenkins, J.M., Haworth, K., Cartwright, S., Morris, R., Rowden, P., Tenenbaum, P., Ting, E.B.: Gravity-darkening analysis of the misaligned hot Jupiter MASCARA-4 b. ApJ 888(2), 63 (2020). https://doi.org/10.3847/1538-4357/ab59d0, arXiv:1911.05025 
4. Akinsanmi, B., Oshagh, M., Santos, N.C., Barros, S.C.C.: Detecting transit signatures of exoplanetary rings using SOAP3.0. A\&A 609, A21 (2018). https://doi.org/10.1051/0004-6361/201731215, arXiv: 1709.06443

5. Akinsanmi, B., Barros, S.C.C., Santos, N.C., Correia, A.C.M., Maxted, P.F.L., Boué, G., Laskar, J.: Detectability of shape deformation in short-period exoplanets. A\&A 621, A117 (2019). https://doi.org/10.1051/0004-6361/201834215, arXiv:1812.04538

6. Albrecht, S., Winn, J.N., Johnson, J.A., Howard, A.W., Marcy, G.W., Butler, R.P., Arriagada, P., Crane, J.D., Shectman, S.A., Thompson, I.B., Hirano, T., Bakos, G., Hartman, J.D.: Obliquities of hot jupiter host stars: evidence for tidal interactions and primordial misalignments. ApJ 757(1), 18 (2012). https://doi.org/10.1088/0004-637X/757/1/18, arXiv:1206.6105

7. Auvergne, M., Bodin, P., Boisnard, L., Buey, J.T., Chaintreuil, S., Epstein, G., Jouret, M., Lam-Trong, T., Levacher, P., Magnan, A., Perez, R., Plasson, P., Plesseria, J., Peter, G., Steller, M., Tiphène, D., Baglin, A., Agogué, P., Appourchaux, T., Barbet, D., Beaufort, T., Bellenger, R., Berlin, R., Bernardi, P., Blouin, D., Boumier, P., Bonneau, F., Briet, R., Butler, B., Cautain, R., Chiavassa, F., Costes, V., Cuvilho, J., Cunha-Parro, V., de Oliveira Fialho, F., Decaudin, M., Defise, J.M., Djalal, S., Docclo, A., Drummond, R., Dupuis, O., Exil, G., Fauré, C., Gaboriaud, A., Gamet, P., Gavalda, P., Grolleau, E., Gueguen, L., Guivarc'h, V., Guterman, P., Hasiba, J., Huntzinger, G., Hustaix, H., Imbert, C., Jeanville, G., Johlander, B., Jorda, L., Journoud, P., Karioty, F., Kerjean, L., Lafond, L., Lapeyrere, V., Landiech, P., Larqué, T., Laudet, P., Le, Merrer. J., Leporati, L., Leruyet, B., Levieuge, B., Llebaria, A., Martin, L., Mazy, E., Mesnager, J.M., Michel, J.P., Moalic, J.P., Monjoin, W., Naudet, D., Neukirchner, S., Nguyen-Kim, K., Ollivier, M., Orcesi, J.L., Ottacher, H., Oulali, A., Parisot, J., Perruchot, S., Piacentino, A., Pinheiro da Silva, L., Platzer, J., Pontet, B., Pradines, A., Quentin, C., Rohbeck, U., Rolland, G., Rollenhagen, F., Romagnan, R., Russ, N., Samadi, R., Schmidt, R., Schwartz, N., Sebbag, I., Smit, H., Sunter, W., Tello, M., Toulouse, P., Ulmer, B., Vandermarcq, O., Vergnault, E., Wallner, R., Waultier, G., Zanatta, P.: The CoRoT satellite in flight: description and performance. A\&A 506(1), 411-424 (2009). https://doi.org/10.1051/0004-6361/200810860, arXiv:0901.2206

8. Bakos, G.Á., Csubry, Z., Penev, K., Bayliss, D., Jordán, A., Afonso, C., Hartman, J.D., Henning, T., Kovács, G., Noyes, R.W., Béky, B., Suc, V., Csák, B., Rabus, M., Lázár, J., Papp, I., Sári, P., Conroy, P., Zhou, G., Sackett, P.D., Schmidt, B., Mancini, L., Sasselov, D.D., Ueltzhoeffer, K.: HATSouth: A global network of fully automated identical wide-field telescopes. PASP 125(924), 154 (2013). https://doi.org/10.1086/669529, arXiv:1206.1391

9. Barnes, J.W.: Transit lightcurves of extrasolar planets orbiting rapidly rotating stars. ApJ 705(1), 683692 (2009). https://doi.org/10.1088/0004-637X/705/1/683, arXiv:0909.1752

10. Barnes, J.W., Fortney, J.J.: Measuring the oblateness and rotation of transiting extrasolar giant planets. ApJ 588(1), 545-556 (2003). https://doi.org/10.1086/373893, arXiv:astro-ph/0301156

11. Barnes, J.W., Linscott, E., Shporer, A.: Measurement of the spin-orbit misalignment of KOI-13.01 from Its gravity-darkened kepler transit lightcurve. ApJS 197(1), 10 (2011). https://doi.org/10.1088/0067-0049/197/1/10, arXiv:1110.3514

12. Benz, W., Broeg, C., Fortier, A., Rando, N., Beck, T., Beck, M., Queloz, D., Ehrenreich, D., Maxted, P.F.L., Isaak, K.G., Billot, N., Alibert, Y., Alonso, R., António, C., Asquier, J., Bandy, T., Bárczy, T., Barrado, D., Barros, S.C.C., Baumjohann, W., Bekkelien, A., Bergomi, M., Biondi, F., Bonfils, X., Borsato, L., Brandeker, A., Busch, M.D., Cabrera, J., Cessa, V., Charnoz, S., Chazelas, B., Collier Cameron, A., Corral Van Damme, C., Cortes, D., Davies, M.B., Deleuil, M., Deline, A., Delrez, L., Demangeon, O., Demory, B.O., Erikson, A., Farinato, J., Fossati, L., Fridlund, M., Futyan, D., Gandolfi, D., Garcia Munoz, A., Gillon, M., Guterman, P., Gutierrez, A., Hasiba, J., Heng, K., Hernandez, E., Hoyer, S., Kiss, L.L., Kovacs, Z., Kuntzer, T., Laskar, J., Lecavelier des Etangs, A., Lendl, M., López, A., Lora, I., Lovis, C., Lüftinger, T., Magrin, D., Malvasio, L., Marafatto, L., Michaelis, H., de Miguel, D., Modrego, D., Munari, M., Nascimbeni, V., Olofsson, G., Ottacher, H., Ottensamer, R., Pagano, I., Palacios, R., Pallé, E., Peter, G., Piazza, D., Piotto, G., Pizarro, A., Pollaco, D., Ragazzoni, R., Ratti, F., Rauer, H., Ribas, I., Rieder, M., Rohlfs, R., Safa, F., Salatti, M., Santos, N.C., Scandariato, G., Ségransan, D., Simon, A.E., Smith, A.M.S., Sordet, M., Sousa, S.G., Steller, M., Szabó, G.M., Szoke, J., Thomas, N., Tschentscher, M., Udry, S., Van, G.rootel.V., Viotto, V., Walter, I., Walton, N.A., Wildi, F., Wolter, D.: The CHEOPS mission. Exper. Astron. https://doi.org/10.1007/s10686-020-09679-4, arXiv:2009.11633 (2020)

13. Borucki, W.J., Koch, D., Basri, G., Batalha, N., Brown, T., Caldwell, D., Caldwell, J., ChristensenDalsgaard, J., Cochran, W.D., DeVore, E., Dunham, E.W., Dupree, A.K., Gautier, T.N., Geary, J.C., 
Gilliland, R., Gould, A., Howell, S.B., Jenkins, J.M., Kondo, Y., Latham, D.W., Marcy, G.W., Meibom, S., Kjeldsen, H., Lissauer, J.J., Monet, D.G., Morrison, D., Sasselov, D., Tarter, J., Boss, A., Brownlee, D., Owen, T., Buzasi, D., Charbonneau, D., Doyle, L., Fortney, J., Ford, E.B., Holman, M.J., Seager, S., Steffen, J.H., Welsh, W.F., Rowe, J., Anderson, H., Buchhave, L., Ciardi, D., Walkowicz, L., Sherry, W., Horch, E., Isaacson, H., Everett, M.E., Fischer, D., Torres, G., Johnson, J.A., Endl, M., MacQueen, P., Bryson, S.T., Dotson, J., Haas, M., Kolodziejczak, J., Van Cleve, J., Chandrasekaran, H., Twicken, J.D., Quintana, E.V., Clarke, B.D., Allen, C., Li, J., Wu, H., Tenenbaum, P., Verner, E., Bruhweiler, F., Barnes, J., Prsa, A.: Kepler planet-detection mission: Introduction and first results. Science 327(5968), 977 (2010). https://doi.org/10.1126/science.1185402

14. Canup, R.M.: Dynamics of lunar formation. ARAA 42(1), 441-475 (2004). https://doi.org/10.1146/ annurev.astro.41.082201.113457

15. Canup, R.M.: Forming a moon with an earth-like composition via a giant impact. Science 338(6110), 1052 (2012). https://doi.org/10.1126/science. 1226073

16. Canup, R.M., Ward, W.R.: Accretion of large satellites of gas planets. In: AAS/Division for Planetary Sciences Meeting Abstracts \#38, AAS/Division for Planetary Sciences Meeting Abstracts, p. 07.02 (2006)

17. Carter, J.A., Winn, J.N.: The detectability of transit depth variations due to exoplanetary oblateness and spin precession. ApJ 716(1), 850-856 (2010). https://doi.org/10.1088/0004-637X/716/1/850, arXiv: 1005.1663

18. Chambers, J.E., Wetherill, G.W.: Making the terrestrial planets: N-body integrations of planetary embryos in three dimensions. Icarus 136(2), 304-327 (1998). https://doi.org/10.1006/icar.1998.6007

19. Charbonneau, D., Brown, T.M., Latham, D.W., Mayor, M.: Detection of planetary transits across a sun-like star. APJL 529(1), L45-L48 (2000). https://doi.org/10.1086/312457, arXiv:astro-ph/9911436

20. Charbonneau, D., Brown, T.M., Noyes, R.W., Gilliland, R.L.: Detection of an extrasolar planet atmosphere. ApJ 568(1), 377-384 (2002). https://doi.org/10.1086/338770, arXiv:astro-ph/0111544

21. Charnoz, S., Morbidelli, A., Dones, L., Salmon, J.: Did Saturn's rings form during the late heavy bombardment? Icarus 199(2), 413-428 (2009). https://doi.org/10.1016/j.icarus.2008.10.019, arXiv:0809.5073

22. Claret A: Comprehensive tables for the interpretation and modeling of the light curves of eclipsing binaries. A\&AS 131, 395-400 (1998). https://doi.org/10.1051/aas:1998278

23. Claret, A.: A new non-linear limb-darkening law for LTE stellar atmosphere models. Calculations for $-5.0 i=\log [\mathrm{M} / \mathrm{H}] i=+1,2000 \mathrm{~K} i=\mathrm{T}_{\text {eff }} i=50000 \mathrm{~K}$ at several surface gravities. A\&A 363, 1081-1190 (2000)

24. Claret, A.: Studies on stellar rotation. II. Gravity-darkening: the effects of the input physics and differential rotation. New results for very low mass stars. A\&A 359, 289-298 (2000)

25. Claret, A.: On the deviations of the classical von Zeipel's theorem at the upper layers of rotating stars. A\&,A 538, A3 (2012). https://doi.org/10.1051/0004-6361/201117419

26. Claret, A.: Limb and gravity-darkening coefficients for the TESS satellite at several metallicities, surface gravities, and microturbulent velocities. A\&A 600, A30 (2017). https://doi.org/10.1051/0004-6361/201629705, arXiv:1804.10295

27. Claret, A.: A new method to compute limb-darkening coefficients for stellar atmosphere models with spherical symmetry: the space missions TESS, Kepler, CoRoT, and MOST. A\&A 618, A20 (2018). https://doi.org/10.1051/0004-6361/201833060, arXiv:1804.10135

28. Claret, A., Hauschildt, P.H., Witte, S.: New limb-darkening coefficients for PHOENIX/1D model atmospheres. I. Calculations for $1500 \mathrm{~K} \leq, \mathrm{T}_{\text {eff }} \leq 4800 \mathrm{~K}$ Kepler, CoRot, Spitzer, uvby, UBVRIJHK, Sloan, and 2MASS photometric systems. A\&A 546, A14 (2012). https://doi.org/10.1051/0004-6361/201219849

29. Claret, A., Hauschildt, P.H., Witte, S.: New limb-darkening coefficients for Sloan, model atmospheres. II. Calculations for $5000 \mathrm{~K} \leq \mathrm{T}_{e f f} \leq 10000 \mathrm{~K}$ Kepler, CoRot, Spitzer, uvby, UBVRIJHK, Sloan, and 2MASS photometric systems. A\&,A 552, A16 (2013). https://doi.org/10.1051/0004-6361/201220942

30. Collier Cameron, A., Wilson, D.M., West, R.G., Hebb, L., Wang, X.B., Aigrain, S., Bouchy, F., Christian, D.J., Clarkson, W.I., Enoch, B., Esposito, M., Guenther, E., Haswell, C.A., Hébrard, G., Hellier, C., Horne, K., Irwin, J., Kane, S.R., Loeillet, B., Lister, T.A., Maxted, P., Mayor, M., Moutou, C., Parley, N., Pollacco, D., Pont, F., Queloz, D., Ryans, R., Skillen, I., Street, R.A., Udry, S., Wheatley, P.J.: Efficient identification of exoplanetary transit candidates from SuperWASP light curves. MNRAS 380(3), 1230-1244 (2007). https://doi.org/10.1111/j.1365-2966.2007.12195.x, arXiv:0707.0417 
31. Collier Cameron A, Guenther, E., Smalley, B., McDonald, I., Hebb, L., Andersen, J., Augusteijn, T., Barros, S.C.C., Brown, D.J.A., Cochran, W.D., Endl, M., Fossey, S.J., Hartmann, M., Maxted, P.F.L., Pollacco, D., Skillen, I., Telting, J., Waldmann, I.P., West, R.G.: Line-profile tomography of exoplanet transits - II. A, gas-giant planet transiting a rapidly rotating A5 star. MNRAS 407(1), 507-514 (2010). https://doi.org/10.1111/j.1365-2966.2010.16922.x, arXiv:1004.4551

32. Correia, A.C.M.: Transit light curve and inner structure of close-in planets. A\&A 570, L5 (2014). https://doi.org/10.1051/0004-6361/201424733, arXiv:1410.5495

33. Coughlin, J., Thompson, S.E., Team Kepler: The final Kepler planet candidate catalog (DR25). In: American Astronomical Society Meeting Abstracts \#230, American Astronomical Society Meeting Abstracts, vol. 230, p. 102.04 (2017)

34. Crida, A., Charnoz, S.: Formation of regular satellites from ancient massive rings in the solar system. Science 338(6111), 1196 (2012). https://doi.org/10.1126/science.1226477, arXiv:1301.3808

35. Crouzet, N., McCullough, P.R., Long, D., Montanes, R.odriguez.P., Lecavelier, d.es.E.tangs.A., Ribas, I., Bourrier, V., Hébrard, G., Vilardell, F., Deleuil, M., Herrero, E., Garcia-Melendo, E., Akhenak, L., Foote, J., Gary, B., Benni, P., Guillot, T., Conjat, M., Mékarnia, D., Garlitz, J., Burke, C.J., Courcol, B., Demangeon, O.: Discovery of XO-6b: A hot jupiter transiting a fast rotating f5 star on an oblique orbit. AJ 153(3), 94 (2017). https://doi.org/10.3847/1538-3881/153/3/94, arXiv:1612.02776

36. Csizmadia, S., Pasternacki, T., Dreyer, C., Cabrera, J., Erikson, A., Rauer, H.: The effect of stellar limb darkening values on the accuracy of the planet radii derived from photometric transit observations. A\&A 549, A9 (2013). https://doi.org/10.1051/0004-6361/201219888, arXiv:1212.2372

37. Deleuil, M., Fridlund, M.: CoRoT: A first space-based transiting survey to explore the close-in planets populations, pp. 1-24. Springer International Publishing, Cham (2018). https://doi.org/10.1007/978-3-319-30648-3-79-1

38. Encrenaz, T., Tinetti, G., Coustenis, A.: Transit spectroscopy of temperate Jupiters with ARIEL: a feasibility study. Exp. Astron. 46(1), 31-44 (2018). https://doi.org/10.1007/s10686-017-9561-2

39. Espinosa Lara, F., Rieutord, M.: Gravity darkening in rotating stars. A\&A 533, A43 (2011). https://doi.org/10.1051/0004-6361/201117252, arXiv:1109.3038

40. Espinoza, N., Jordán, A.: Limb darkening and exoplanets: testing stellar model atmospheres and identifying biases in transit parameters. MNRAS 450(2), 1879-1899 (2015). https://doi.org/10.1093/mnras/stv744, arXiv:1503.07020

41. Espinoza, N., Jordán, A.: Limb darkening and exoplanets - II. Choosing the best law for optimal retrieval of transit parameters. MNRAS 457(4), 3573-3581 (2016). https://doi.org/10.1093/mnras/stw224, arXiv:1601.05485

42. Faigler, S., Mazeh, T.: Photometric detection of non-transiting short-period low-mass companions through the beaming, ellipsoidal and reflection effects in Kepler and CoRoT light curves. MNRAS 415(4), 3921-3928 (2011). https://doi.org/10.1111/j.1365-2966.2011.19011.x, arXiv:1106.2713

43. Galland, F., Lagrange, A.M., Udry, S., Chelli, A., Pepe, F., Queloz, D., Beuzit, J.L., Mayor, M.: Extrasolar planets and brown dwarfs around A-F type stars. I. Performances of radial velocity measurements, first analyses of variations. A\&A 443(1), 337-345 (2005). https://doi.org/10.1051/0004-6361:20052938, arXiv:astro-ph/0509111

44. Heller, R., Rodenbeck, K., Bruno, G.: An alternative interpretation of the exomoon candidate signal in the combined Kepler and Hubble data of Kepler-1625. A\&A 624, A95 (2019). https://doi.org/10.1051/0004-6361/201834913, arXiv:1902.06018

45. Holman, M.J., Murray, N.W.: The use of transit timing to detect terrestrial-mass extrasolar planets. Science 307(5713), 1288-1291 (2005). https://doi.org/10.1126/science.1107822, arXiv:astro-ph/0412028

46. Howarth, I.D.: On stellar limb darkening and exoplanetary transits. MNRAS 418(2), 1165-1175 (2011). https://doi.org/10.1111/j.1365-2966.2011.19568.x, arXiv:1106.4659

47. Johnson, M.C., Rodriguez, J.E., Zhou, G., Gonzales, E.J., Cargile, P.A., Crepp, J.R., Penev, K., Stassun, K.G., Gaudi, B.S., Colón, K.D., Stevens, D.J., Strassmeier, K.G., Ilyin, I., Collins, K.A., Kielkopf, J.F., Oberst, T.E., Maritch, L., Reed, P.A., Gregorio, J., Bozza, V., Calchi, N.ovati.S., D’Ago, G., Scarpetta, G., Zambelli, R., Latham, D.W., Bieryla, A., Cochran, W.D., Endl, M., Tayar, J., Serenelli, A., Silva, A.guirre.V., Clarke, S.P., Martinez, M., Spencer, M., Trump, J., Joner, M.D., Bugg, A.G., Hintz, E.G., Stephens, D.C., Arredondo, A., Benzaid, A., Yazdi, S., McLeod, K.K., Jensen, E.L.N., Hancock, D.A., Sorber, R.L., Kasper, D.H., Jang-Condell, H., Beatty, T.G., Carroll, T., Eastman, J., James, D., Kuhn, R.B., Labadie-Bartz, J., Lund, M.B., Mallonn, M., Pepper, J., Siverd, R.J., Yao, X., Cohen, D.H., Curtis, I.A., DePoy, D.L., Fulton, B.J., Penny, M.T., Relles, H., Stockdale, C., Tan, T.G., 
Villanueva, J.S.: KELT-21b: A hot jupiter transiting the rapidly rotating metal-poor late-a primary of a likely hierarchical triple system. AJ 155(2), 100 (2018). https://doi.org/10.3847/1538-3881/aaa5af, arXiv: 1712.03241

48. Kaula, W.M.: Theory of satellite geodesy. Applications of satellites to geodesy (1966)

49. Kipping, D.M.: Transit timing effects due to an exomoon. MNRAS 392(1), 181-189 (2009). https://doi.org/10.1111/j.1365-2966.2008.13999.x, arXiv:0810.2243

50. Kipping, D.M.: Transit timing effects due to an exomoon - II. MNRAS 396(3), 1797-1804 (2009). https://doi.org/10.1111/j.1365-2966.2009.14869.x, arXiv:0904.2565

51. Kipping, D.M., Bakos, G.Á., Buchhave, L., Nesvorný, D., Schmitt, A.: The hunt for Exomoons with Kepler (HEK). I. Description of a New Observational project. ApJ 750(2), 115 (2012). https://doi.org/10.1088/0004-637X/750/2/115, arXiv:1201.0752

52. Kraft, R.P.: Studies of stellar rotation. V. The Dependence of rotation on age among solar-type stars. ApJ 150, 551 (1967). https://doi.org/10.1086/149359

53. Lendl, M., Csizmadia, S., Deline, A., Fossati, L., Kitzmann, D., Heng, K., Hoyer, S., Salmon, S., Benz, W., Broeg, C., Ehrenreich, D., Fortier, A., Queloz, D., Bonfanti, A., Brandeker, A., Collier Cameron, A., Delrez, L., Garcia Muñoz, A., Hooton, M.J., Maxted, P.F.L., Morris, B.M., Van Grootel, V., Wilson, T.G., Alibert, Y., Alonso, R., Asquier, J., Bandy, T., Bárczy, T., Barrado, D., Barros, S.C.C., Baumjohann, W., Beck, M., Beck, T., Bekkelien, A., Bergomi, M., Billot, N., Biondi, F., Bonfils, X., Bourrier, V., Busch, M.D., Cabrera, J., Cessa, V., Charnoz, S., Chazelas, B., Corral Van Damme, C., Davies, M.B., Deleuil, M., Demangeon, O.D.S., Demory, B.O., Erikson, A., Farinato, J., Fridlund, M., Futyan, D., Gandolfi, D., Gillon, M., Guterman, P., Hasiba, J., Hernandez, E., Isaak, K.G., Kiss, L., Kuntzer, T., Lecavelier des Etangs, A., Lüftinger, T., Laskar, J., Lovis, C., Magrin, D., Malvasio, L., Marafatto, L., Michaelis, H., Munari, M., Nascimbeni, V., Olofsson, G., Ottacher, H., Ottensamer, R., Pagano, I., Pallé, E., Peter, G., Piazza, D., Piotto, G., Pollacco, D., Ratti, F., Rauer, H., Ragazzoni, R., Rando, N., Ribas, I., Rieder, M., Rohlfs, R., Safa, F., Santos, N.C., Scandariato, G., Ségransan, D., Simon, A.E., Singh, V., Smith, A.M.S., Sordet, M., Sousa, S.G., Steller, M., Szabó, G.M., Thomas, N., Tschentscher, M., Udry, S., Viotto, V., Walter, I., Walton, N.A., Wildi, F., Wolter, D.: The hot dayside and asymmetric transit of WASP-189 b seen by CHEOPS. A\&A 643, A94 (2020). https://doi.org/10.1051/0004-6361/202038677, arXiv:2009.13403

54. Mandel, K., Agol, E.: Analytic light curves for planetary transit searches. APJL 580(2), L171-L175 (2002). https://doi.org/10.1086/345520, arXiv:astro-ph/0210099

55. Maxted, P.F.L.: Comparison of the power-2 limb-darkening law from the STAGGERgrid to Kepler light curves of transiting exoplanets. A\&A 616, A39 (2018). https://doi.org/10.1051/0004-6361/201832944, arXiv:1804.07943

56. Miralda-Escudé, J.: Orbital perturbations of transiting planets: a possible method to measure stellar quadrupoles and to detect earth-mass planets. ApJ 564(2), 1019-1023 (2002). https://doi.org/10.1086/324279, arXiv:astro-ph/0104034

57. Morbidelli, A., Tsiganis, K., Batygin, K., Crida, A., Gomes, R.: Explaining why the uranian satellites have equatorial prograde orbits despite the large planetary obliquity. Icarus 219(2), 737-740 (2012). https://doi.org/10.1016/j.icarus.2012.03.025, arXiv:1208.4685

58. Morello, G., Claret, A., Martin-Lagarde, M., Cossou, C., Tsiaras, A., Lagage, P.O.: The ExoTETHyS package: Tools for exoplanetary transits around host stars. AJ 159(2), 75 (2020). https://doi.org/10.3847/1538-3881/ab63dc, arXiv:1908.09599

59. Mugnai, L.V., Pascale, E., Edwards, B., Papageorgiou, A., Sarkar, S.: ArielRad: the Ariel radiometric model. Exper. Astron. 50(2-3), 303-328 (2020). https://doi.org/10.1007/s10686-020-09676-7, arXiv:2009.07824

60. Nagasawa, M., Thommes, E.W., Kenyon, S.J., Bromley, B.C., Lin, D.N.C.: The diverse origins of terrestrial-planet systems. In: Reipurth, B., Jewitt, D., Keil, K. (eds.) Protostars and planets V, p. 639 (2007)

61. Pál, A.: Properties of analytic transit light-curve models. MNRAS 390(1), 281-288 (2008). https://doi.org/10.1111/j.1365-2966.2008.13723.x, arXiv:0805.2157

62. Pál, A.: FITSH- a software package for image processing. MNRAS 421(3), 1825-1837 (2012). https://doi.org/10.1111/j.1365-2966.2011.19813.x, arXiv:1111.1998

63. Pascale, E., Bezawada, N., Barstow, J., Beaulieu, J.P., Bowles, N., Coudé du Foresto, V., Coustenis, A., Decin, L., Drossart, P., Eccleston, P., Encrenaz, T., Forget, F., Griffin, M., Güdel, M., Hartogh, P., Heske, A., Lagage, P.O., Leconte, J., Malaguti, P., Micela, G., Middleton, K., Min, M., Moneti, A., Morales, J.C., Mugnai, L., Ollivier, M., Pace, E., Papageorgiou, A., Pilbratt, G., Puig, L., Rataj, 
M., Ray, T., Ribas, I., Rocchetto, M., Sarkar, S., Selsis, F., Taylor, W., Tennyson, J., Tinetti, G., Turrini, D., Vandenbussche, B., Venot, O., Waldmann, I.P., Wolkenberg, P., Wright, G., Zapatero Osorio, M.R., Zingales, T.: The ARIEL space mission. In: Lystrup, M., MacEwen, H.A., Fazio, G.G., Batalha, N., Siegler, N., Tong, E.C. (eds.) Space Telescopes and Instrumentation 2018: Optical, Infrared, and Millimeter Wave, Society of Photo-Optical Instrumentation Engineers (SPIE) Conference Series, vol. 10698, p. 106980H (2018). https://doi.org/10.1117/12.2311838

64. Pilbratt, G.: ARIEL: ESA's mission to study the nature of exoplanets. In: AAS/Division for Extreme Solar Systems Abstracts, AAS/Division for Extreme Solar Systems Abstracts, vol. 51, p. 503.04 (2019)

65. Pollacco, D.L., Skillen, I., Collier Cameron, A., Christian, D.J., Hellier, C., Irwin, J., Lister, T.A., Street, R.A., West, R.G., Anderson, D.R., Clarkson, W.I., Deeg, H., Enoch, B., Evans, A., Fitzsimmons, A., Haswell, C.A., Hodgkin, S., Horne, K., Kane, S.R., Keenan, F.P., Maxted, P.F.L., Norton, A.J., Osborne, J., Parley, N.R., Ryans, R.S.I., Smalley, B., Wheatley, P.J., Wilson, D.M.: The WASP project and the SuperWASP cameras. PASP 118(848), 1407-1418 (2006). https://doi.org/10.1086/508556, arXiv:astro-ph/0608454

66. Puig, L., Pilbratt, G., Heske, A., Escudero, I., Crouzet, P.E., de Vogeleer, B., Symonds, K., Kohley, R., Drossart, P., Eccleston, P., Hartogh, P., Leconte, J., Micela, G., Ollivier, M., Tinetti, G., Turrini, D., Vandenbussche, B., Wolkenberg, P.: The phase A study of the ESA M4 mission candidate ARIEL. Exp. Astron. 46(1), 211-239 (2018). https://doi.org/10.1007/s10686-018-9604-3

67. Ricker, G.R., Winn, J.N., Vanderspek, R., Latham, D.W., Bakos, G.Á., Bean, J.L., Berta-Thompson, Z.K., Brown, T.M., Buchhave, L., Butler, N.R., Butler, R.P., Chaplin, W.J., Charbonneau, D., Christensen-Dalsgaard, J., Clampin, M., Deming, D., Doty, J., De Lee, N., Dressing, C., Dunham, E.W., Endl, M., Fressin, F., Ge, J., Henning, T., Holman, M.J., Howard, A.W., Ida, S., Jenkins, J.M., Jernigan, G., Johnson, J.A., Kaltenegger, L., Kawai, N., Kjeldsen, H., Laughlin, G., Levine, A.M., Lin, D., Lissauer, J.J., MacQueen, P., Marcy, G., McCullough, P.R., Morton, T.D., Narita, N., Paegert, M., Palle, E., Pepe, F., Pepper, J., Quirrenbach, A., Rinehart, S.A., Sasselov, D., Sato, B., Seager, S., Sozzetti, A., Stassun, K.G., Sullivan, P., Szentgyorgyi, A., Torres, G., Udry, S., Villasenor, J.: Transiting exoplanet survey satellite (TESS). J. Astron. Telescopes Instrum. Syst. 1, 014003 (2015). https://doi.org/10.1117/1.JATIS.1.1.014003

68. Rosich, A., Herrero, E., Mallonn, M., Ribas, I., Morales, J.C., Perger, M., Anglada-Escudé, G., Granzer, T.: Correcting for chromatic stellar activity effects in transits with multiband photometric monitoring: application to WASP-52. A\&A 641, A82 (2020). https://doi.org/10.1051/0004-6361/ 202037586, arXiv:2007.00573

69. Rozelot, J.P., Neiner, C.: The rotation of sun and stars, vol 765. https://doi.org/10.1007/978-3-54087831-5 (2009)

70. Sarkar, S., Pascale, E., Papageorgiou, A., Johnson, L.J., Waldmann, I.: ExoSim: the Exoplanet Observation Simulator. arXiv:2002.03739 (2020)

71. Sasaki, T., Stewart, G.R., Ida, S.: Origin of the Different Architectures of the Jovian and Saturnian Satellite Systems. ApJ 714(2), 1052-1064 (2010). https://doi.org/10.1088/0004-637X/714/2/1052, arXiv: 1003.5737

72. Seager, S.: Exoplanets (2010)

73. Seager, S., Hui, L.: Constraining the rotation rate of transiting extrasolar planets by oblateness measurements. ApJ 574(2), 1004-1010 (2002). https://doi.org/10.1086/340994, arXiv:astro-ph/0204225

74. Shporer, A.: The astrophysics of visible-light orbital phase curves in the space age. PASP 129(977), 072001 (2017). https://doi.org/10.1088/1538-3873/aa7112, arXiv:1703.00496

75. Shporer, A., O’Rourke, J.G., Knutson, H.A., Szabó, G.M., Zhao, M., Burrows, A., Fortney, J., Agol, E., Cowan, N.B., Desert, J.M., Howard, A.W., Isaacson, H., Lewis, N.K., Showman, A.P., Todorov, K.O.: Atmospheric characterization of the hot jupiter kepler-13Ab. ApJ 788(1), 92 (2014). https://doi.org/10.1088/0004-637X/788/1/92, arXiv:1403.6831

76. Simon, A., Szatmáry, K., Szabó, G.M.: Determination of the size, mass, and density of "exomoons" from photometric transit timing variations. A\&A 470(2), 727-731 (2007). https://doi.org/10.1051/0004-6361:20066560, arXiv:0705.1046

77. Simon, A.E., Szabó, G.M., Szatmáry, K., Kiss, L.L.: Methods for exomoon characterization: combining transit photometry and the Rossiter-McLaughlin effect. MNRAS 406(3), 2038-2046 (2010). https://doi.org/10.1111/j.1365-2966.2010.16818.x, arXiv:1004.1143 
78. Szabó, G.M., Szatmáry, K., Divéki, Z., Simon, A.: Possibility of a photometric detection of "exomoons". A\&A 450(1), 395-398 (2006). https://doi.org/10.1051/0004-6361:20054555, arXiv:astro-ph/0601186

79. Szabó, G.M., Szabó, R., Benkő, J.M., Lehmann, H., Mező, G., Simon, A.E., Kővári, Z., Hodosán, G., Regály, Z., Kiss, L.L.: Asymmetric transit curves as indication of orbital obliquity: clues from the late-type dwarf companion in KOI-13. APJL 736(1), L4 (2011). https://doi.org/10.1088/2041-8205/736/1/L4, arXiv:1105.2524

80. Szabó, G.M., Pál, A., Derekas, A., Simon, A.E., Szalai, T., Kiss, L.L.: Spin-orbit resonance, transit duration variation and possible secular perturbations in KOI-13. MNRAS 421(1), L122-L126 (2012). https://doi.org/10.1111/j.1745-3933.2012.01219.x, arXiv:1110.4231

81. Szabó, G.M., Pribulla, T., Pál, A., Bódi, A., Kiss, L.L., Derekas, A.: The clockwork is moving on a combined analysis of TESS and Kepler measurements of Kepler-13Ab. MNRAS 492(1), L17-L21 (2020). https://doi.org/10.1093/mnrasl/slz177, arXiv:1911.11399

82. Szabó, R., Szabó, G.M., Dálya, G., Simon, A.E., Hodosán, G., Kiss, L.L.: Multiple planets or exomoons in Kepler hot Jupiter systems with transit timing variations? A\&A 553, A17 (2013). https://doi.org/10.1051/0004-6361/201220132, arXiv:1207.7229

83. Teachey, A., Kipping, D.M.: Evidence for a large exomoon orbiting Kepler-1625b. Sci. Adv. 4(10), eaav1784 (2018). https://doi.org/10.1126/sciadv.aav1784, arXiv:1810.02362

84. Tinetti, G., Drossart, P., Eccleston, P., Hartogh, P., Heske, A., Leconte, J., Micela, G., Ollivier, M., Pilbratt, G., Puig, L., et al.: A chemical survey of exoplanets with ARIEL. Exp. Astron. 46(1), 135-209 (2018). https://doi.org/10.1007/s10686-018-9598-x

85. Triaud, A.H.M.J., Collier Cameron, A., Queloz, D., Anderson, D.R., Gillon, M., Hebb, L., Hellier, C., Loeillet, B., Maxted, P.F.L., Mayor, M., Pepe, F., Pollacco, D., Ségransan, D., Smalley, B., Udry, S., West, R.G., Wheatley, P.J.: Spin-orbit angle measurements for six southern transiting planets. New insights into the dynamical origins of hot Jupiters. A\&A 524, A25 (2010). https://doi.org/10.1051/0004-6361/201014525, arXiv:1008.2353

86. Tusnski, L.R.M., Valio, A.: Transit model of planets with moon and ring systems. ApJ 743(1), 97 (2011). https://doi.org/10.1088/0004-637X/743/1/97, arXiv:1111.5599

87. van Saders, J.L., Pinsonneault, M.H.: Fast star, slow star; old star, young star: subgiant rotation as a population and stellar physics diagnostic. ApJ 776(2), 67 (2013). https://doi.org/10.1088/0004-637X/776/2/67, arXiv:1306.3701

88. von Zeipel, H.: The radiative equilibrium of a rotating system of gaseous masses. MNRAS 84, 665683 (1924). https://doi.org/10.1093/mnras/84.9.665

89. Watanabe, N., Narita, N., Johnson, M.C.: Doppler tomographic measurement of the nodal precession of WASP-33b. PASJ. https://doi.org/10.1093/pasj/psz140, arXiv:2003.02724 (2020)

90. Winn, J.N.: Transits and occultations. arXiv:1001.2010 (2010)

91. Zingales, T., Tinetti, G., Pillitteri, I., Leconte, J., Micela, G., Sarkar, S.: The ARIEL mission reference sample. Exper. Astron. 46(1), 67-100 (2018). https://doi.org/10.1007/s10686-018-9572-7, arXiv: 1706.08444

92. Zuluaga, J.I., Kipping, D.M., Sucerquia, M., Alvarado, J.A.: A novel method for identifying exoplanetary rings. APJL 803(1), L14 (2015). https://doi.org/10.1088/2041-8205/803/1/L14, arXiv: 1502.07818

Publisher's note Springer Nature remains neutral with regard to jurisdictional claims in published maps and institutional affiliations. 


\section{Affiliations}

\section{Gyula M. Szabó ${ }^{1,2}$ (D) . Szilárd Kálmán ${ }^{1,2,3}$. Theodor Pribulla ${ }^{2,4}$. Antonio Claret $^{5,6}$. Lorenzo V. Mugnai ${ }^{7}$. Enzo Pascale ${ }^{7}$. Dave Waltham ${ }^{8}$. Luca Borsato $^{9} \cdot$ Zoltán Garai $^{2,4} \cdot$ Róbert Szabó $^{10,11,12}$}

1 MTA-ELTE Exoplanet Research Group, Gothard Astrophysical Observatory, ELTE Eötvös Loránd University, Szent Imre h. u. 112, Szombathely, Hungary

2 MTA-ELTE Exoplanet Research Group, Szent Imre h. u. 112, Szombathely, Hungary

3 Institute of Physics, University of Szeged, Dóm tér 9, Szeged, Hungary

4 Astronomical Institute of the Slovak Academy of Sciences, 05960 Tatranská Lomnica, Slovakia

5 CSIC, Instituto de Astrofísica de Andalucía, Apartado 3004, 18080 Granada, Spain

6 Department of Física Teórica y del Cosmos, Universidad de Granada, Campus de Fuentenueva s/n, 10871, Granada, Spain

7 Dipartimento di Fisica, La Sapienza Università di Roma, Roma, Italy

8 Department of Earth Sciences, University of London, Royal Holloway, UK

9 INAF-Osservatorio Astronomico di Padova, Vicolo dell'Osservatorio 5, 35122, Padova, Italy

10 CSFK, ELKH, Konkoly Observatory, Konkoly T. M. u. 17, Budapest, Hungary

11 Institute of Physics, ELTE Eötvös Loránd University, Pázmány P. s. 1A, Budapest, Hungary

12 MTA CSFK Lendület Near-Field Cosmology Research Group, Konkoly T. M. u. 17, Budapest, Hungary 